Supporting Information for

\title{
Species Prioritization Based on Spectral Dissimilarity: A Case Study of Polyporoid Fungal
} Species

Huong T. Pham, ${ }^{\dagger}$ Kwang Ho Lee, ${ }^{\ddagger}$ Eunah Jeong, ${ }^{\dagger}$ Sunmin Woo, ${ }^{\dagger}, \S$ Jinsuh Yu, ${ }^{\dagger}$ Woo-Young Kim, ${ }^{\dagger}$ Young Woon Lim,^ Ki Hyun Kim,,,** and Kyo Bin Kang ${ }^{\dagger, *}$

${ }^{\dagger}$ Research Institute of Pharmaceutical Sciences, College of Pharmacy, Sookmyung Women's University, Seoul 04310, Korea

‡ School of Pharmacy, Sungkyunkwan University, Suwon 16419, Korea

$\S$ College of Pharmacy and Research Institute of Pharmaceutical Sciences, Seoul National University, Seoul 08826, Korea

$\wedge$ School of Biological Sciences and Institute of Microbiology, Seoul National University, Seoul 08826, Korea

${ }^{*}$ Corresponding authors.

E-mail: khkim83@skku.edu (K. H. Kim); kbkang@sookmyung.ac.kr (K. B. Kang) 


\section{Table of Contents}

Figure S1. The feature-based molecular network of 40 Polyporaceae fungal culture extracts. The colored nine candidate species were selected based on the PCoA plot (Figure 1A).

Figure S2. Schematic overview on how sample occurrence per molecular family was calculated. 'Component index' is an index for each molecular family, which can be retrieved from GNPS molecular networking job results. These examples are from the Polyporaceae molecular network, which is shown in Figure S1.

Figure S3. The molecular network of the fungal culture extract (red color) and fruiting bodies extract (blue color) of $C$. volvatus showed that most spectral nodes in the fruiting bodies extract are clustered together with the major peaks of the mycelial extract, which implies their structural similarity. The BPI chromatogram of the fungal culture extract is attached to visualize pairs between major chromatographic peaks and spectral nodes.

Figure S4. The entire feature-based molecular network of the C. volvatus fruiting bodies extract and fractions. The cryptoporic acid molecular family, which is highlighted in the main text and Figure 3, is marked with a red box.

Figure S5. MM2-optimized 3D structural model of compounds 2 (A) and 4 (B).

Table S1. Detailed parameters used for Mzmine2 preprocessing.

Table S2. ${ }^{1} \mathrm{H}$ and ${ }^{13} \mathrm{C}$ NMR Spectroscopic Data of Compounds 5-9 (500/125 MHz, in $\left.\mathrm{CDCl}_{3}\right)$

Table S3. Cytotoxicity of the Isolated Triterpenoids against HCT-116 Cells.

Figure S6. ${ }^{1} \mathrm{H}$ NMR Spectrum of Cryptoporic Acid T (1) (500 MHz, in $\left.\mathrm{CDCl}_{3}\right)$.

Figure S7. ${ }^{13} \mathrm{C}$ NMR Spectrum of Cryptoporic Acid T (1) (125 MHz, in $\left.\mathrm{CDCl}_{3}\right)$.

Figure S8. ${ }^{1} \mathrm{H}-{ }^{1} \mathrm{H}$ COSY Spectrum of Cryptoporic Acid T (1) (500 MHz, in $\left.\mathrm{CDCl}_{3}\right)$.

Figure S9. Multiplicity-edited HSQC Spectrum of Cryptoporic Acid T (1) (500 MHz, in CDCl $)$.

Figure S10. HMBC Spectrum of Cryptoporic Acid T (1) (500 MHz, in $\left.\mathrm{CDCl}_{3}\right)$.

Figure S11. ROESY Spectrum of Cryptoporic Acid T (1) (500 MHz, in $\left.\mathrm{CDCl}_{3}\right)$.

Figure S12. ${ }^{1} \mathrm{H}$ NMR Spectrum of 5"', 6"'-cryptoporic acid G dimethyl ester (2) (500 MHz, in $\mathrm{CDCl}_{3}$ ). 
Figure S13. ${ }^{13} \mathrm{C}$ NMR Spectrum of 5"', 6"'-cryptoporic acid G dimethyl ester (2) (125 MHz, in $\mathrm{CDCl}_{3}$ ).

Figure S14. ${ }^{1} \mathrm{H}-{ }^{1} \mathrm{H}$ COSY Spectrum of 5"',6"'-cryptoporic acid G dimethyl ester (2) (500 MHz, in $\left.\mathrm{CDCl}_{3}\right)$.

Figure S15. Multiplicity-edited HSQC Spectrum of 5"', 6"'-cryptoporic acid G dimethyl ester (2) (500 MHz, in $\mathrm{CDCl}_{3}$ ).

Figure S16. HMBC Spectrum of 5"',6"'-cryptoporic acid G dimethyl ester (2) (500 MHz, in $\left.\mathrm{CDCl}_{3}\right)$.

Figure S17. ROESY Spectrum of 5"',6"'-cryptoporic acid G dimethyl ester (2) (500 MHz, in $\left.\mathrm{CDCl}_{3}\right)$.

Figure S18. ${ }^{1} \mathrm{H}$ NMR Spectrum of 5"-cryptoporic acid E isopropyl ester (3) (500 MHz, in $\mathrm{CDCl}_{3}$ ).

Figure S19. ${ }^{13} \mathrm{C}$ NMR Spectrum of 5"-cryptoporic acid E isopropyl ester (3) (125 MHz, in $\left.\mathrm{CDCl}_{3}\right)$.

Figure S20. ${ }^{1} \mathrm{H}-{ }^{1} \mathrm{H}$ COSY Spectrum of 5"-cryptoporic acid E isopropyl ester (3) (500 MHz, in $\left.\mathrm{CDCl}_{3}\right)$.

Figure S21. Multiplicity-edited HSQC Spectrum of 5"-cryptoporic acid E isopropyl ester (3) $\left(500 \mathrm{MHz}\right.$, in $\left.\mathrm{CDCl}_{3}\right)$.

Figure S22. HMBC Spectrum of 5"-cryptoporic acid E isopropyl ester (3) (500 MHz, in $\mathrm{CDCl}_{3}$ ).

Figure S23. ROESY Spectrum of 5"-cryptoporic acid E isopropyl ester (3) (500 MHz, in $\left.\mathrm{CDCl}_{3}\right)$.

Figure S24. ${ }^{1} \mathrm{H}$ NMR Spectrum of Cryptoporic acid D trimethyl ester (4) (500 MHz, in MeOD).

Figure S25. ${ }^{13} \mathrm{C}$ NMR Spectrum of Cryptoporic acid D trimethyl ester (4) (125 MHz, in MeOD).

Figure S26. ${ }^{1} \mathrm{H}-{ }^{1} \mathrm{H}$ COSY Spectrum of Cryptoporic acid D trimethyl ester (4) (500 MHz, in $\mathrm{MeOD})$.

Figure S27. Multiplicity-edited HSQC Spectrum of Cryptoporic acid D trimethyl ester (4) (500 $\mathrm{MHz}$, in MeOD).

Figure S28. HMBC Spectrum of Cryptoporic acid D trimethyl ester (4) (500 MHz, in MeOD). 


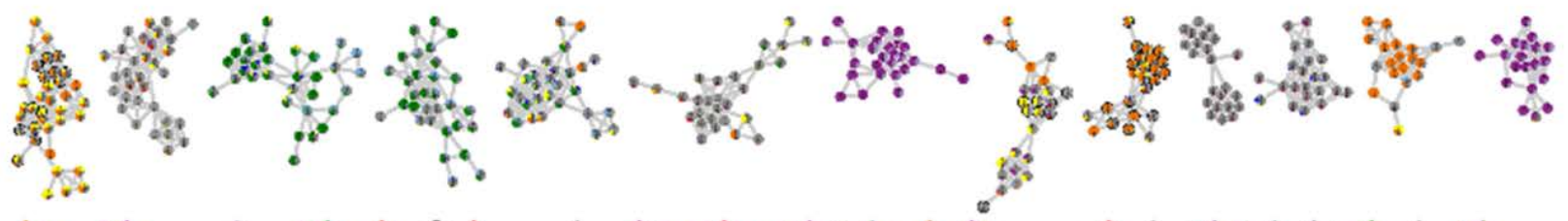

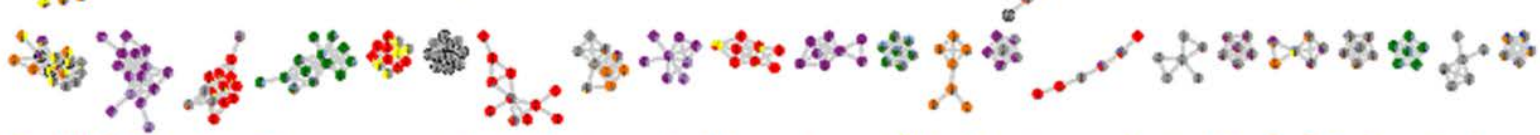

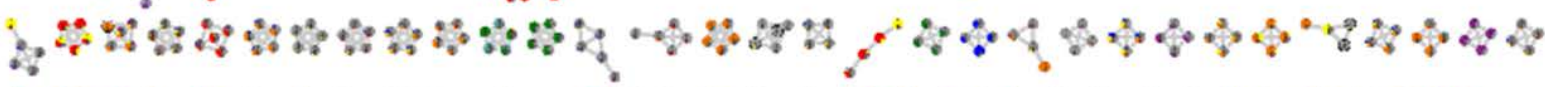

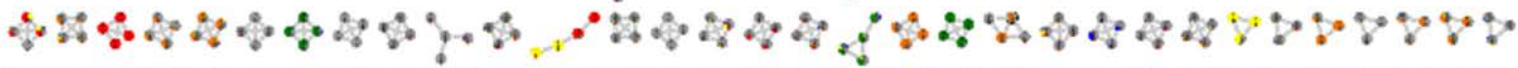

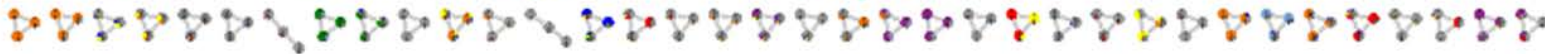

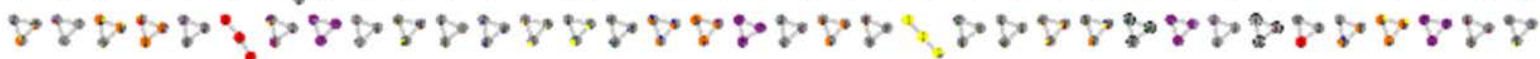

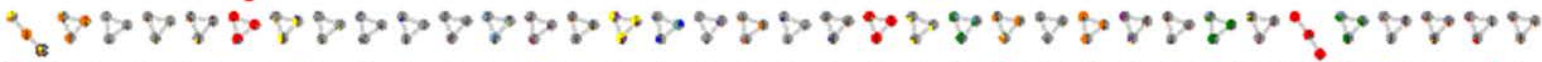

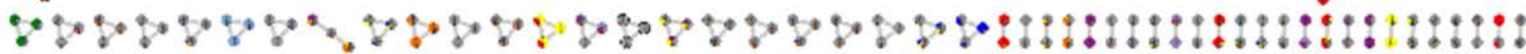

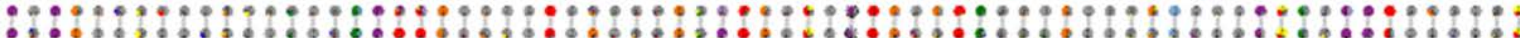
†

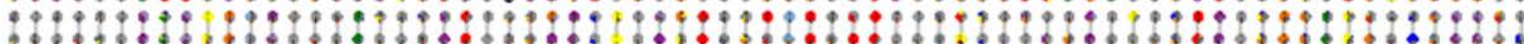
: : : : : *

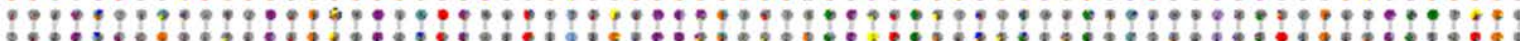

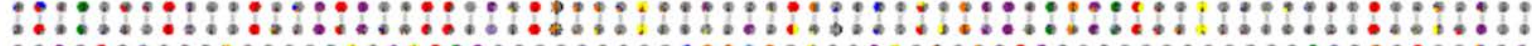
$\because: 0_{0}$ : 0

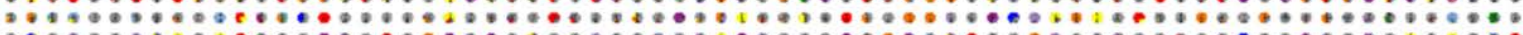
$\because 40 ; 0_{0}$ (a) (a) $\because \ldots$ :

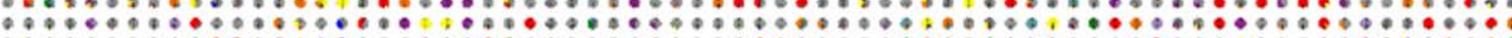

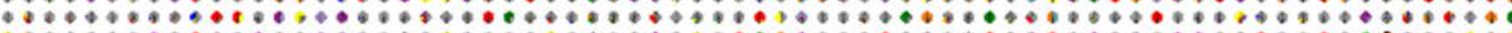
 $\because: \because z_{0}$ O

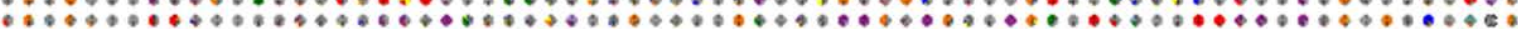
$\bullet_{\bullet}$

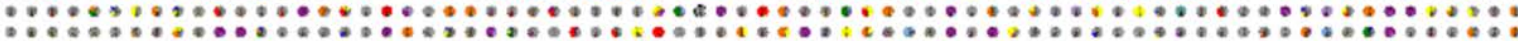

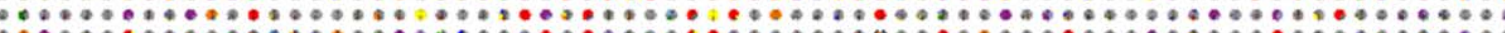
0 : $\because$ 0 н

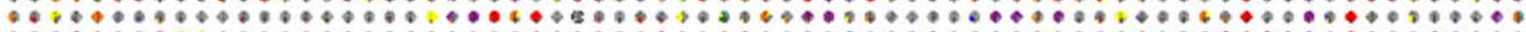

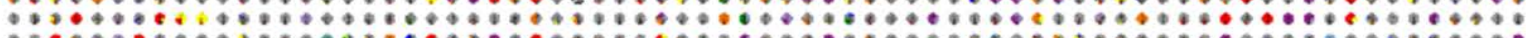
-

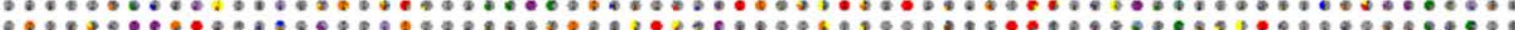
(a) : $\because \cdots$

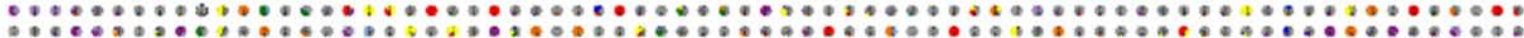
$\because: \because:: \because: \because::$

\section{Color legend}

\begin{tabular}{|c|c|c|c|c|}
\hline $\begin{array}{l}\text { Cryptoporus volvatus } \\
\text { (KMRB16041501) }\end{array}$ & $\begin{array}{l}\text { Phlebiopsis gigantea } \\
\text { (KMRB17083132) }\end{array}$ & $\begin{array}{l}\text { Daedaleopsis tricolor } \\
\text { (KMRB15061217) }\end{array}$ & $\begin{array}{l}\text { Polyporus brumalis } \\
\text { (KMRB15102011) }\end{array}$ & $\begin{array}{l}\text { Microporus vernicipes } \\
\text { (KMRB15071608) }\end{array}$ \\
\hline $\begin{array}{l}\text { Tyromyces chioneus } \\
\text { (KMRB14070116) }\end{array}$ & $\begin{array}{l}\text { Skeletocutis nivea } \\
\text { (KMRB15090322) }\end{array}$ & $\begin{array}{l}\text { Lopharia cinerascens } \\
\text { (KMRB15090836) }\end{array}$ & $\begin{array}{l}\text { Tramates versicolor } \\
\text { (KMRB15032003) }\end{array}$ & the other species \\
\hline
\end{tabular}

Figure S1. The feature-based molecular network of 40 Polyporaceae fungi culture extracts. The colored nine candidate species were selected based on the PCoA plot (Figure 1A). 
(A)

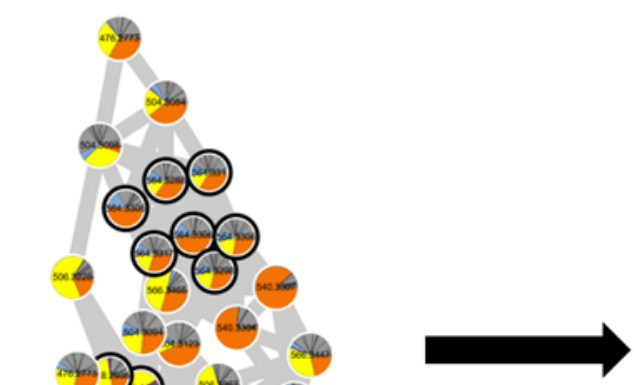

Every spectra in this molecular families found from all the 40 species but Daedaleopsis confragosa and Perenniporia koreana

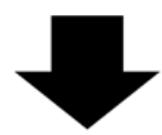

Sample occurrence $=38$

(component index 16)

(B)

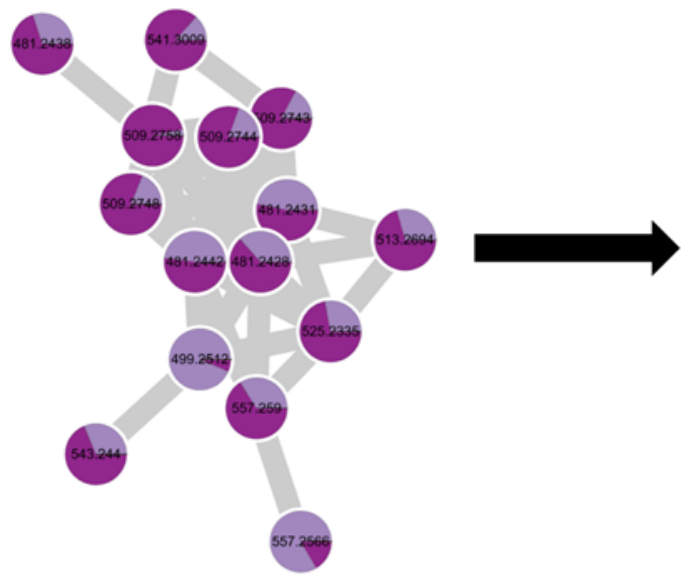

Every spectra in this molecular families found only from Tyromyces chioneus $(\bigcirc)$ and Skeletoticus nivea

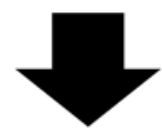

\section{Sample occurrence $=2$}

(component index 293)

Figure S2. Schematic overview on how sample occurrence per molecular family was calculated. 'Component index' is an index for each molecular family, which can be retrieved from GNPS molecular networking job results. These examples are from the Polyporaceae molecular network, which is shown in Figure S1. 


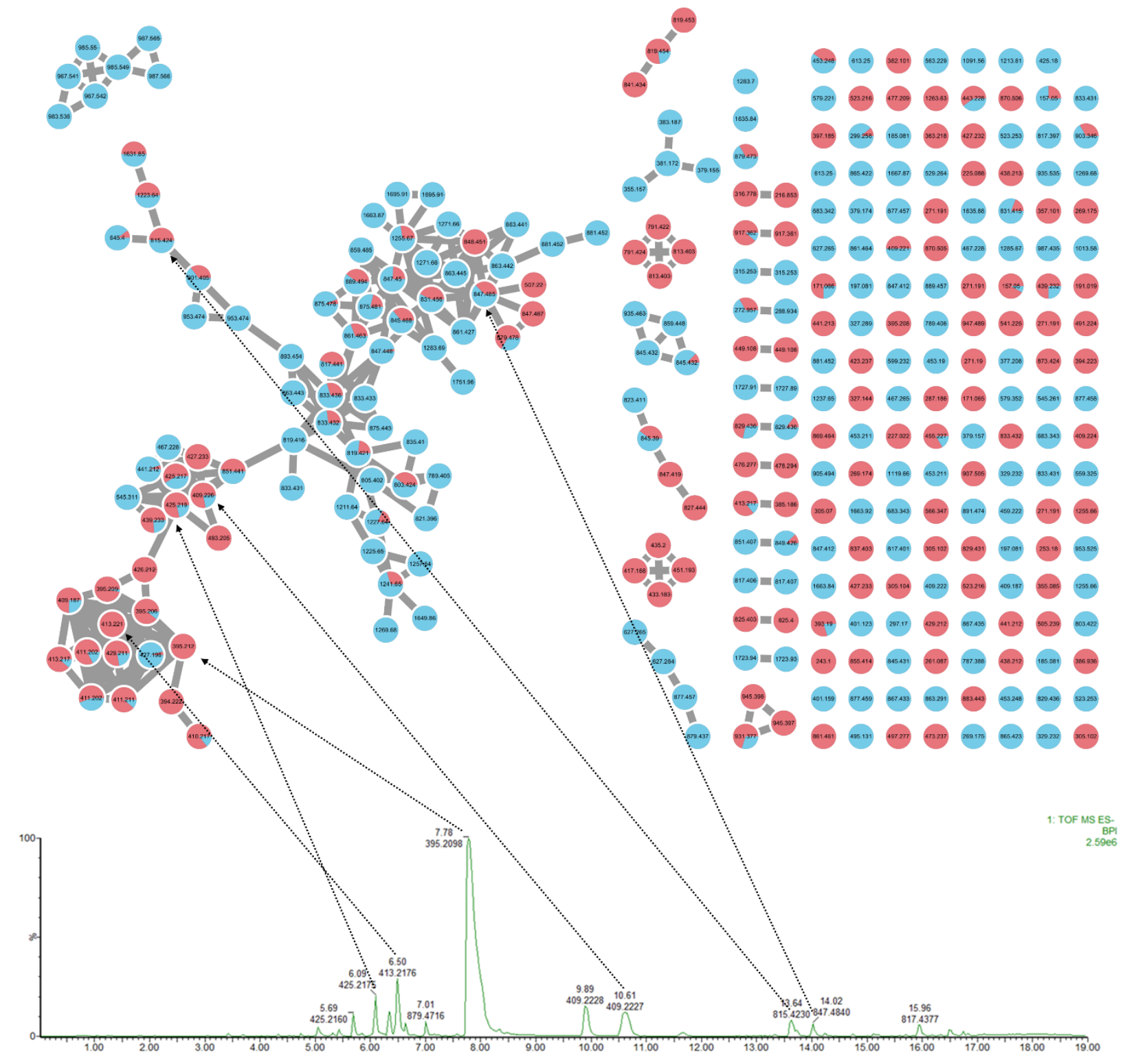

Figure S3. The molecular network of the fungal culture extract (red color) and fruiting bodies extract (blue color) of $C$. volvatus showed that most spectral nodes in the fruiting bodies extract are clustered together with the major peaks of the mycelial extract, which implies their structural similarity. The BPI chromatogram of the fungal culture extract is attached to visualize pairs between major chromatographic peaks and spectral nodes. 


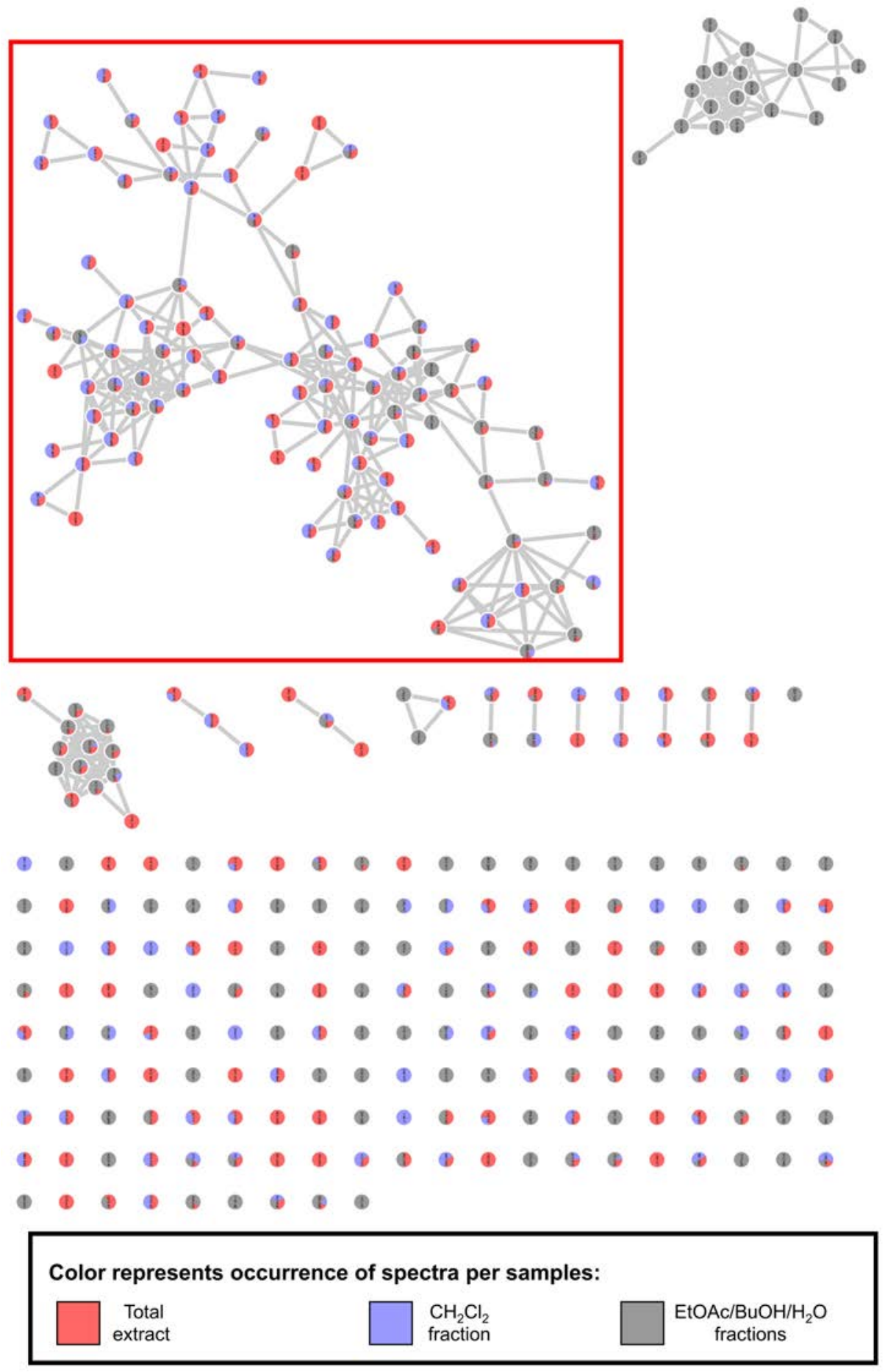

Figure S4. The entire feature-based molecular network of the C. volvatus fruiting bodies extract and fractions. The cryptoporic acid molecular family, which is highlighted in the main text and Figure 3, is marked with a red box. 


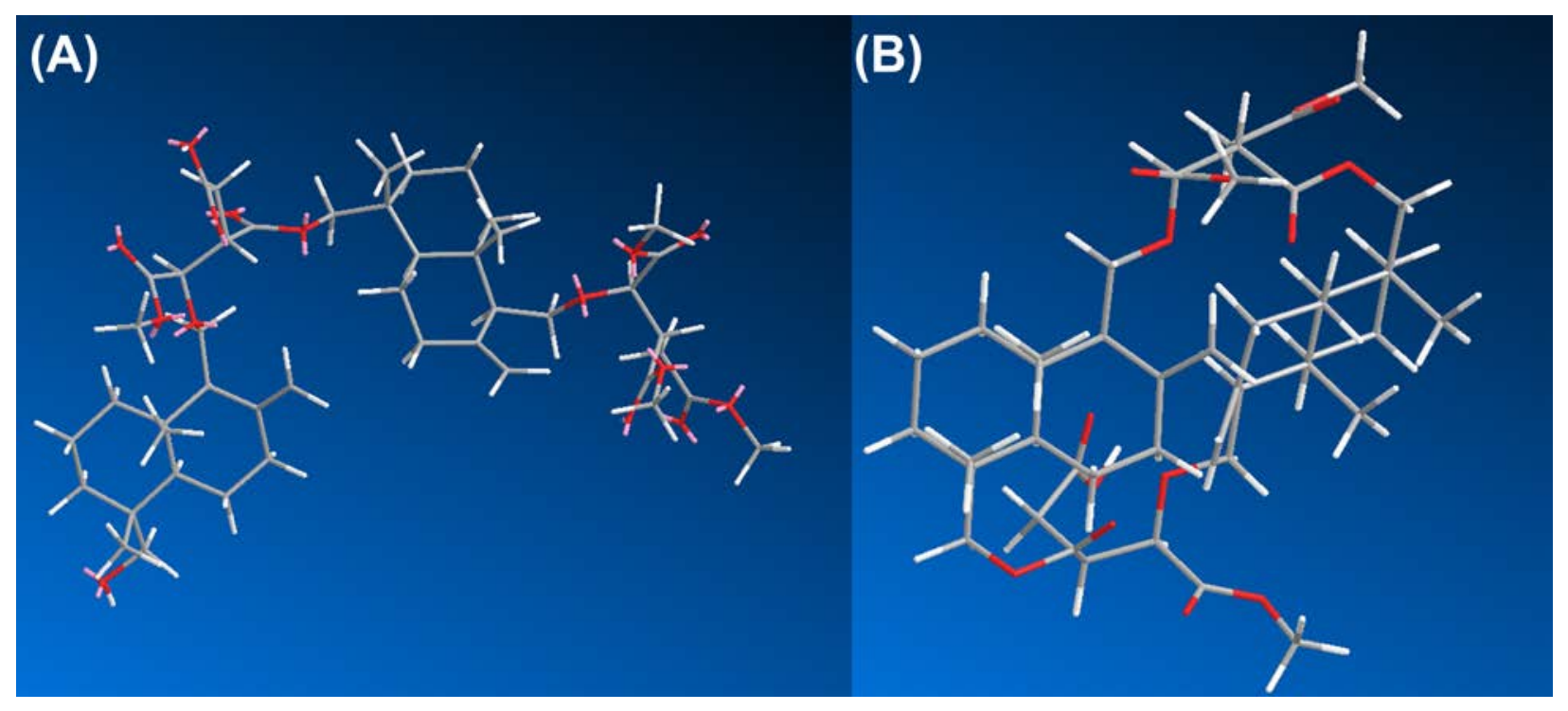

Figure S5. MM2-optimized 3D structural model of compounds 2 (A) and 4 (B). 
Table S1. Detailed parameters used for Mzmine2 preprocessing.

\begin{tabular}{|l|l|l|}
\hline \multicolumn{1}{|c|}{ processing module } & \multicolumn{2}{|c|}{ parameters } \\
\hline peak detection & $\begin{array}{l}\text { noise level for MS1 } \\
\text { noise level for MS2 }\end{array}$ & $0.0 \mathrm{E} 2$ \\
\hline ADAP chromatogram builder & $\begin{array}{l}\text { min group size } \\
\text { group intensity threshold } \\
\text { min highest intensity } \\
m / z \text { tolerance }\end{array}$ & $3.0 \mathrm{E} 2$ \\
& min peak height & $1.0 \mathrm{E} 3$ \\
\hline (baseline-cutoff) & peak duration range & 0.002 Da or $10 \mathrm{ppm}$ \\
\hline isotopic peak grouper & baseline level & $3.0 \mathrm{E} 2$ \\
& $m / z$ tolerance & $0.01-3.00 \mathrm{~min}$ \\
& $\mathrm{t}_{\mathrm{R}}$ tolerance & $1.0 \mathrm{E} 2$ \\
\hline max charge & $0.002 \mathrm{Da}$ or $10 \mathrm{ppm}$ \\
\hline group MS2 scans with features & $\mathrm{t}_{\mathrm{R}}$ tolerance & $0.1 \mathrm{~min}$ \\
& $\mathrm{~m} / \mathrm{z}$ tolerance & 2 \\
\hline join aligner & $\mathrm{t}_{\mathrm{R}}$ tolerance (50 \% weighted) & $0.15 \mathrm{~min}$ \\
& $\mathrm{~m} / \mathrm{z}$ tolerance (50\% weighted) & $0.002 \mathrm{Da}$ or $10 \mathrm{ppm}$ \\
\hline duplicate peak filter & $\mathrm{t}_{\mathrm{R}}$ tolerance (50 \% weighted) & $0.15 \mathrm{~min}$ \\
& $\mathrm{~m} / \mathrm{z}$ tolerance (50\% weighted) & $0.005 \mathrm{Da}$ or $20 \mathrm{ppm}$ \\
\hline gap-Filling & intensity tolerance & $20 \%$ \\
& $\mathrm{t}_{\mathrm{R}}$ tolerance & $0.15 \mathrm{~min}$ \\
& $\mathrm{~m} / \mathrm{z}$ tolerance & $0.002 \mathrm{Da}$ or $10 \mathrm{ppm}$ \\
\hline
\end{tabular}


Table S2. ${ }^{1} \mathrm{H}$ and ${ }^{13} \mathrm{C}$ NMR Spectroscopic Data of Compounds 5-9 (500/125 MHz, in $\left.\mathrm{CDCl}_{3}\right)$

\begin{tabular}{|c|c|c|c|c|c|c|c|c|c|c|}
\hline \multirow{2}{*}{ position } & \multicolumn{2}{|c|}{5} & \multicolumn{2}{|c|}{6} & \multicolumn{2}{|c|}{7} & \multicolumn{2}{|c|}{8} & \multicolumn{2}{|c|}{9} \\
\hline & $\delta_{C}$ & $\delta_{\mathrm{H}}(\mathrm{J}$ in $\mathrm{Hz})$ & $\delta_{\mathrm{C}}$ & $\delta_{\mathrm{H}}(\mathrm{J}$ in $\mathrm{Hz})$ & $\delta_{\mathrm{C}}$ & $\delta_{\mathrm{H}}(\mathrm{J}$ in $\mathrm{Hz})$ & $\delta_{\mathrm{C}}$ & $\delta_{\mathrm{H}}(J$ in $\mathrm{Hz})$ & $\delta_{\mathrm{C}}$ & $\delta_{\mathrm{H}}(\mathrm{J}$ in $\mathrm{Hz})$ \\
\hline \multirow[t]{2}{*}{ A1 } & \multirow[t]{2}{*}{38.6} & $\begin{array}{c}1.66, \mathrm{dd} \\
(12.6,2.0)\end{array}$ & \multirow[t]{2}{*}{38.8} & & \multirow[t]{2}{*}{38.9} & $1.68, \mathrm{~m}$ & \multirow[t]{2}{*}{38.8} & & \multirow[t]{2}{*}{39.3} & \\
\hline & & $\begin{array}{c}\text { 1.15, ddd } \\
(23.3,12.6 \text {, } \\
4.5)\end{array}$ & & & & $\begin{array}{c}1.17, \mathrm{td} \\
(12.3,4.9)\end{array}$ & & & & \\
\hline A2 & 18.5 & $1.56, \mathrm{~m}$ & 18.6 & & 18.5 & $1.56, \mathrm{~m}$ & 18.6 & & 19.3 & \\
\hline \multirow[t]{2}{*}{ A3 } & \multirow[t]{2}{*}{35.4} & $2.80, \mathrm{~m}$ & \multirow[t]{2}{*}{35.3} & & \multirow[t]{2}{*}{35.4} & $1.44, \mathrm{~m}$ & \multirow[t]{2}{*}{35.6} & & \multirow[t]{2}{*}{42.1} & \\
\hline & & $1.45, \mathrm{~m}$ & & & & $1.29, \mathrm{~m}$ & & & & \\
\hline A4 & 38.0 & & 38.0 & & 38.0 & & 37.2 & & 33.6 & \\
\hline A5 & 48.0 & $1.43, \mathrm{~m}$ & 48.1 & & 48.3 & $1.45, \mathrm{~m}$ & 46.5 & & 55.7 & \\
\hline \multirow[t]{2}{*}{ A6 } & \multirow[t]{2}{*}{23.7} & $1.57, \mathrm{~m}$ & \multirow[t]{2}{*}{23.4} & & \multirow[t]{2}{*}{23.7} & $1.60, \mathrm{~m}$ & \multirow[t]{2}{*}{23.3} & & \multirow[t]{2}{*}{24} & $\begin{array}{c}1.10, \mathrm{dd} \\
(12.6,2.6)\end{array}$ \\
\hline & & $1.33, \mathrm{~m}$ & & & & $1.34, \mathrm{~m}$ & & & & \\
\hline \multirow[t]{2}{*}{ A7 } & \multirow[t]{2}{*}{37.3} & $2.35, \mathrm{~m}$ & \multirow[t]{2}{*}{37.1} & $\begin{array}{c}2.35, \mathrm{dd} \\
(10.9,4.4)\end{array}$ & \multirow[t]{2}{*}{37.4} & $2.35, \mathrm{~m}$ & \multirow[t]{2}{*}{37.5} & 2.19, d (13.1) & \multirow[t]{2}{*}{37.7} & $\begin{array}{c}\text { 2.37, ddd } \\
(13.1,4.2 \text {, } \\
2.2)\end{array}$ \\
\hline & & $2.01, \mathrm{~m}$ & & $1.94, \mathrm{~m}$ & & $2.02, \mathrm{~m}$ & & & & $\begin{array}{c}2.02, \mathrm{td} \\
(13.1,4.9)\end{array}$ \\
\hline A8 & 146.5 & & 146.3 & & 146.5 & & 146.4 & $1.92, \mathrm{~m}$ & 146.9 & \\
\hline A9 & 55.9 & $1.98, \mathrm{~m}$ & 55.7 & 1.84, d (8.1) & 55.7 & 1.98, m & 57.2 & & 55.2 & 1.94, d (5.7) \\
\hline A10 & 38.9 & & 38.4 & & 38.7 & & 38.3 & $1.92, \mathrm{~m}$ & 38.8 & \\
\hline \multirow[t]{2}{*}{ A11 } & \multirow[t]{2}{*}{68.5} & $3.94, \mathrm{~m}$ & \multirow[t]{2}{*}{68.5} & $\begin{array}{c}3.88, \mathrm{dd} \\
(9.5,8.3)\end{array}$ & 68.5 & $3.92, \mathrm{~m}$ & 69.7 & $4.13, \mathrm{t}$ & 68.4 & $\begin{array}{c}3.90, \mathrm{dd} \\
(9.7,8.5)\end{array}$ \\
\hline & & $3.52, \mathrm{~m}$ & & $3.57, \mathrm{~m}$ & & $3.53, \mathrm{~m}$ & & $\begin{array}{c}3.36, \mathrm{dd} \\
(9.1,2.4)\end{array}$ & & $\begin{array}{c}3.53, \text { dd } \\
(9.7,3.6)\end{array}$ \\
\hline A12 & 108.7 & $4.88, \mathrm{~s}$ & 108.1 & $4.84, \mathrm{~s}$ & 108.4 & 4.85, br s & 109.0 & 4.97, s & 108.1 & $4.85, \mathrm{~s}$ \\
\hline & & 4.77, br s & & $4.71, \mathrm{~s}$ & & 4.77, d (1.2) & & $4.83, \mathrm{~s}$ & & $4.75, \mathrm{~s}$ \\
\hline A13 & 17.8 & $0.76, \mathrm{~s}$ & 15.9 & $0.73, \mathrm{~s}$ & 16.0 & $0.76, \mathrm{~s}$ & 16.0 & $0.74, \mathrm{~s}$ & 15.5 & $0.72, \mathrm{~s}$ \\
\hline
\end{tabular}




\begin{tabular}{|c|c|c|c|c|c|c|c|c|c|c|}
\hline A14 & 16.0 & $0.75, \mathrm{~s}$ & 17.7 & $0.75, \mathrm{~s}$ & 17.6 & $0.75, \mathrm{~s}$ & 18.0 & $0.69, \mathrm{~s}$ & 21.9 & $0.80, \mathrm{~s}$ \\
\hline \multirow[t]{2}{*}{ A15 } & \multirow[t]{2}{*}{72.0} & $3.43, \mathrm{~m}$ & \multirow[t]{2}{*}{71.2} & 3.40, d (10.9) & \multirow[t]{2}{*}{72.2} & $3.40, \mathrm{~m}$ & \multirow[t]{2}{*}{71.3} & 4.53, d (11.1) & \multirow[t]{2}{*}{33.8} & $0.87, \mathrm{~s}$ \\
\hline & & 3.09, d (11.0) & & 3.08, d (10.9) & & 3.10, d (10.9) & & 2.99, m & & \\
\hline A1' & 79.0 & $4.07, \mathrm{~d}(4.5)$ & 77.3 & $4.11, \mathrm{~m}$ & 78.7 & 4.09, d (4.6) & 79.2 & 3.99, d (3.5) & 78.4 & 4.12, d (4.8) \\
\hline A2' & 44.8 & $3.51, \mathrm{~m}$ & 43.6 & 3.46, m & 44.8 & 3.46, m & 44.8 & 3.58, td & 44.4 & $\begin{array}{c}3.40, \mathrm{dt} \\
(9.3,4.9)\end{array}$ \\
\hline \multirow[t]{2}{*}{ A3' } & \multirow[t]{2}{*}{32.4} & $\begin{array}{c}\text { 2.83, dd } \\
(17.2,4.7)\end{array}$ & \multirow[t]{2}{*}{32.3} & $2.79, \mathrm{~m}$ & \multirow[t]{2}{*}{32.1} & 2.79, t (9.3) & \multirow[t]{2}{*}{32.9} & $2.98, \mathrm{~m}$ & \multirow[t]{2}{*}{31.7} & $\begin{array}{c}2.81, \mathrm{dd} \\
(17.4,9.3)\end{array}$ \\
\hline & & $\begin{array}{c}2.57, \mathrm{dd} \\
(17.2,5.0)\end{array}$ & & $\begin{array}{c}2.61, \mathrm{dd} \\
(17.6,2.7)\end{array}$ & & $2.55, \mathrm{t}(5.3)$ & & $\begin{array}{c}2.75, \text { dd } \\
(16.7,6.3)\end{array}$ & & $\begin{array}{c}2.63, \text { dd } \\
(17.4,4.9)\end{array}$ \\
\hline A $44^{\prime}$ & 171.2 & & 171.0 & & 171.4 & & 171.5 & & 171 & \\
\hline A5' & 170.8 & & 170.9 & & 171.0 & & 169.3 & & 171.3 & \\
\hline A6' & 172.8 & & 178.0 & & 172.4 & & 177.5 & & 177.6 & \\
\hline A4'-OMe & 52.3 & $3.77, \mathrm{~s}$ & 52.5 & $3.67, \mathrm{~s}$ & 52.1 & $3.68, \mathrm{~s}$ & 52.2 & $3.78, \mathrm{~s}$ & $52.2^{\mathrm{a}}$ & $3.69, \mathrm{~s}$ \\
\hline A6'-OMe & 52.3 & $3.70, \mathrm{~s}$ & 52.3 & $3.74, \mathrm{~s}$ & 52.2 & $3.679, \mathrm{~s}$ & & & $52.5^{\mathrm{a}}$ & $3.75, \mathrm{~s}$ \\
\hline \multirow[t]{2}{*}{ B1 } & \multirow[t]{2}{*}{38.5} & $\begin{array}{c}1.66, \mathrm{dd} \\
(11.9,2.0)\end{array}$ & \multirow[t]{2}{*}{38.3} & & \multirow[t]{2}{*}{38.7} & $1.68, \mathrm{~m}$ & & & & \\
\hline & & $\begin{array}{c}\text { 1.15, ddd } \\
(23.3,11.9 \text {, } \\
4.5)\end{array}$ & & & & $\begin{array}{c}1.17, \mathrm{td} \\
(12.3,4.9)\end{array}$ & & & & \\
\hline B2 & 18.6 & 1.56, m & 18.4 & & 18.7 & $1.56, \mathrm{~m}$ & & & & \\
\hline \multirow[t]{2}{*}{ B3 } & \multirow[t]{2}{*}{35.7} & $1.40, \mathrm{~m}$ & \multirow[t]{2}{*}{35.3} & & \multirow[t]{2}{*}{35.8} & 1.36, m & & & & \\
\hline & & 1.30, m & & & & & & & & \\
\hline B4 & 37.0 & & 37.7 & & 36.9 & & & & & \\
\hline B5 & 48.4 & 1.34, m & 46.8 & & 49.1 & $1.34, \mathrm{~m}$ & & & & \\
\hline \multirow[t]{2}{*}{ B6 } & \multirow[t]{2}{*}{23.7} & 1.57, m & \multirow[t]{2}{*}{23.7} & & \multirow[t]{2}{*}{23.9} & $1.55, \mathrm{~m}$ & & & & \\
\hline & & 1.33, m & & & & 1.34, m & & & & \\
\hline \multirow[t]{2}{*}{ B7 } & \multirow[t]{2}{*}{37.4} & 2.37, m & \multirow[t]{2}{*}{37.4} & $\begin{array}{c}2.35, \mathrm{dd} \\
(10.9,4.4)\end{array}$ & \multirow[t]{2}{*}{37.5} & 2.37, m & & & & \\
\hline & & 2.04, m & & 1.94, m & & $2.04, \mathrm{~m}$ & & & & \\
\hline B8 & 146.3 & & 146.6 & & 146.6 & & & & & \\
\hline
\end{tabular}




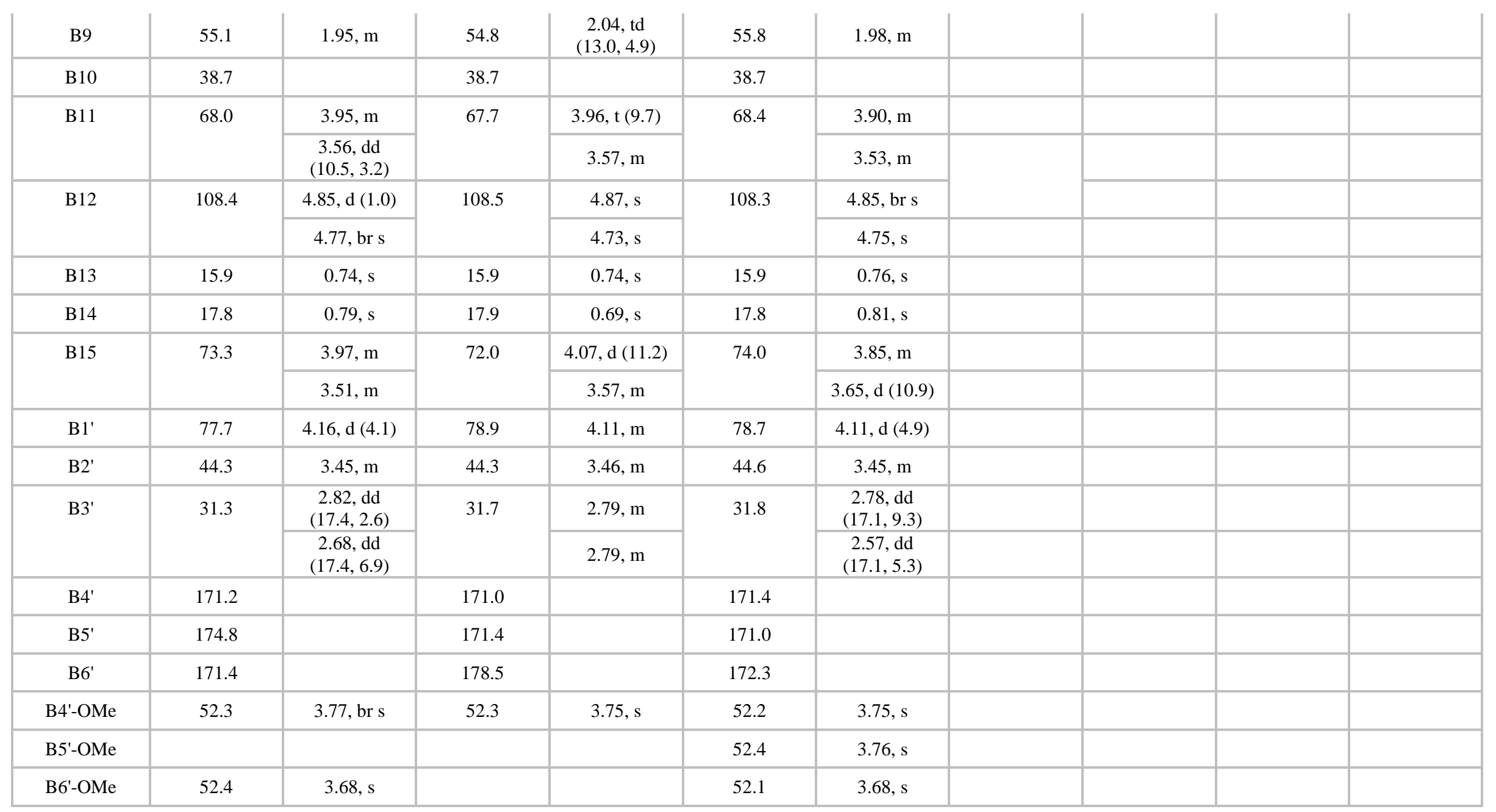

a exchangeable 
Table S3. Cytotoxicity of the Isolated Triterpenoids against HCT-116 Cells. ${ }^{a}$

\begin{tabular}{ccc}
\hline compound & IC $_{50}$ & 95\% confidence interval \\
\hline $\mathbf{2}$ & $4.3 \mu \mathrm{M}$ & $3.7-5.0 \mu \mathrm{M}$ \\
$\mathbf{5}$ & $3.6 \mu \mathrm{M}$ & $3.3-3.8 \mu \mathrm{M}$ \\
paclitaxel $^{b}$ & $7.7 \mathrm{nM}$ & $3.7-15.8 \mathrm{nM}$
\end{tabular}

${ }^{\mathrm{a}}$ Compounds 1, 3, 4, and 6-9 showed $\mathrm{IC}_{50}$ values of $>10 \mu \mathrm{M} .{ }^{\mathrm{b}}$ Positive control. 


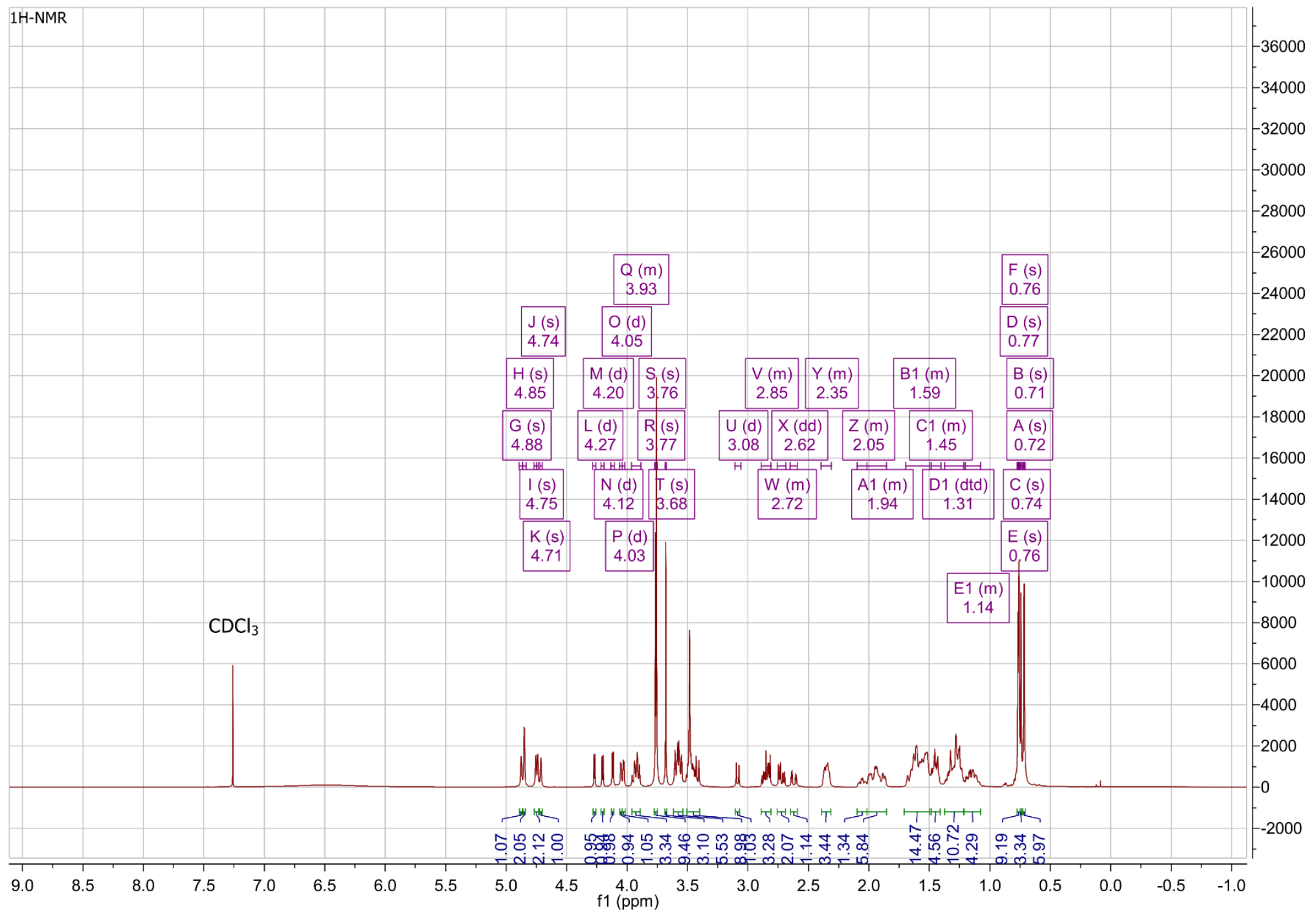

Figure S6. ${ }^{1} \mathrm{H}$ NMR Spectrum of Cryptoporic Acid T (1) (500 MHz, in $\mathrm{CDCl}_{3}$ ). 


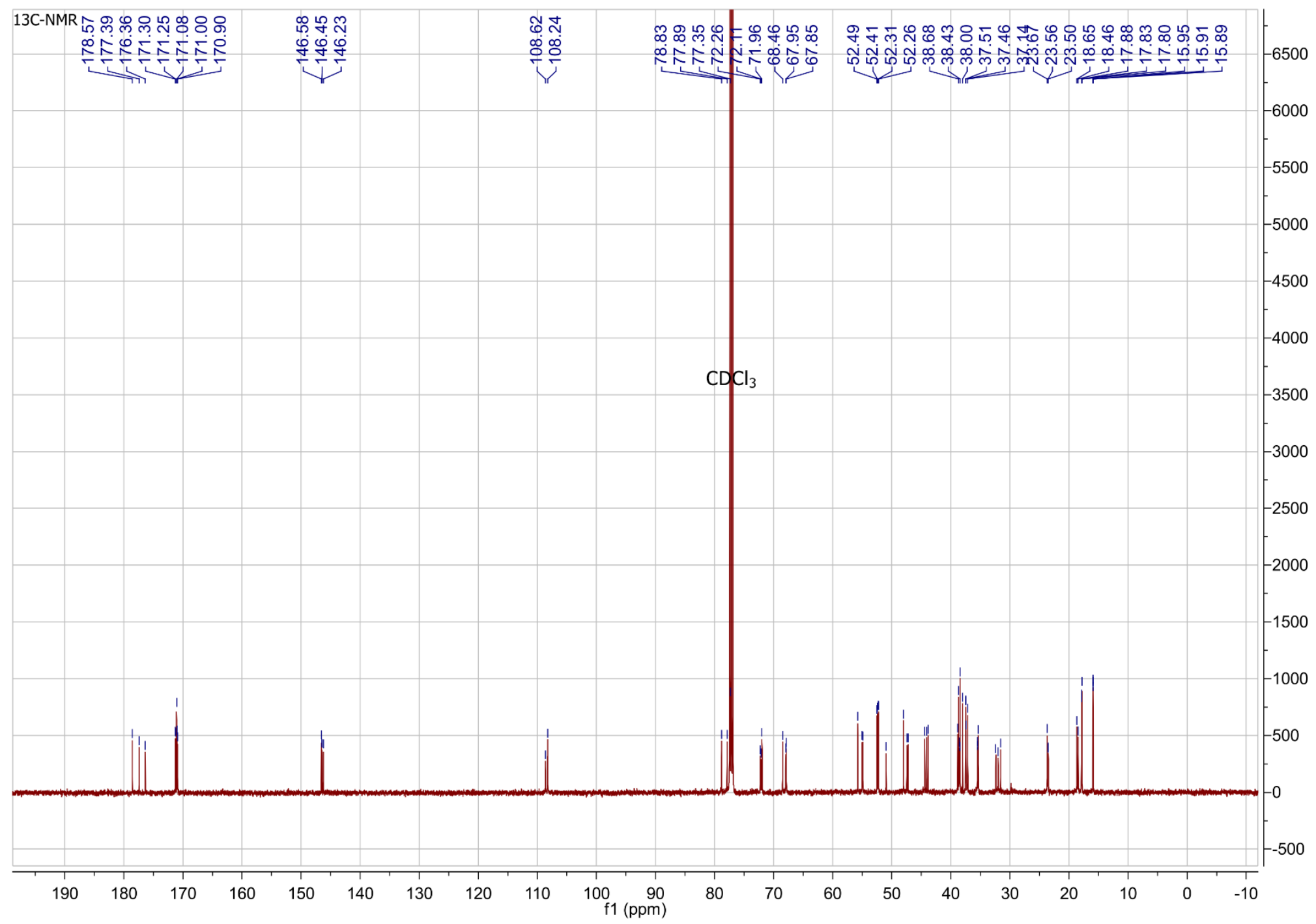

Figure S7. ${ }^{13} \mathrm{C}$ NMR Spectrum of Cryptoporic Acid T (1) (125 MHz, in $\left.\mathrm{CDCl}_{3}\right)$. 


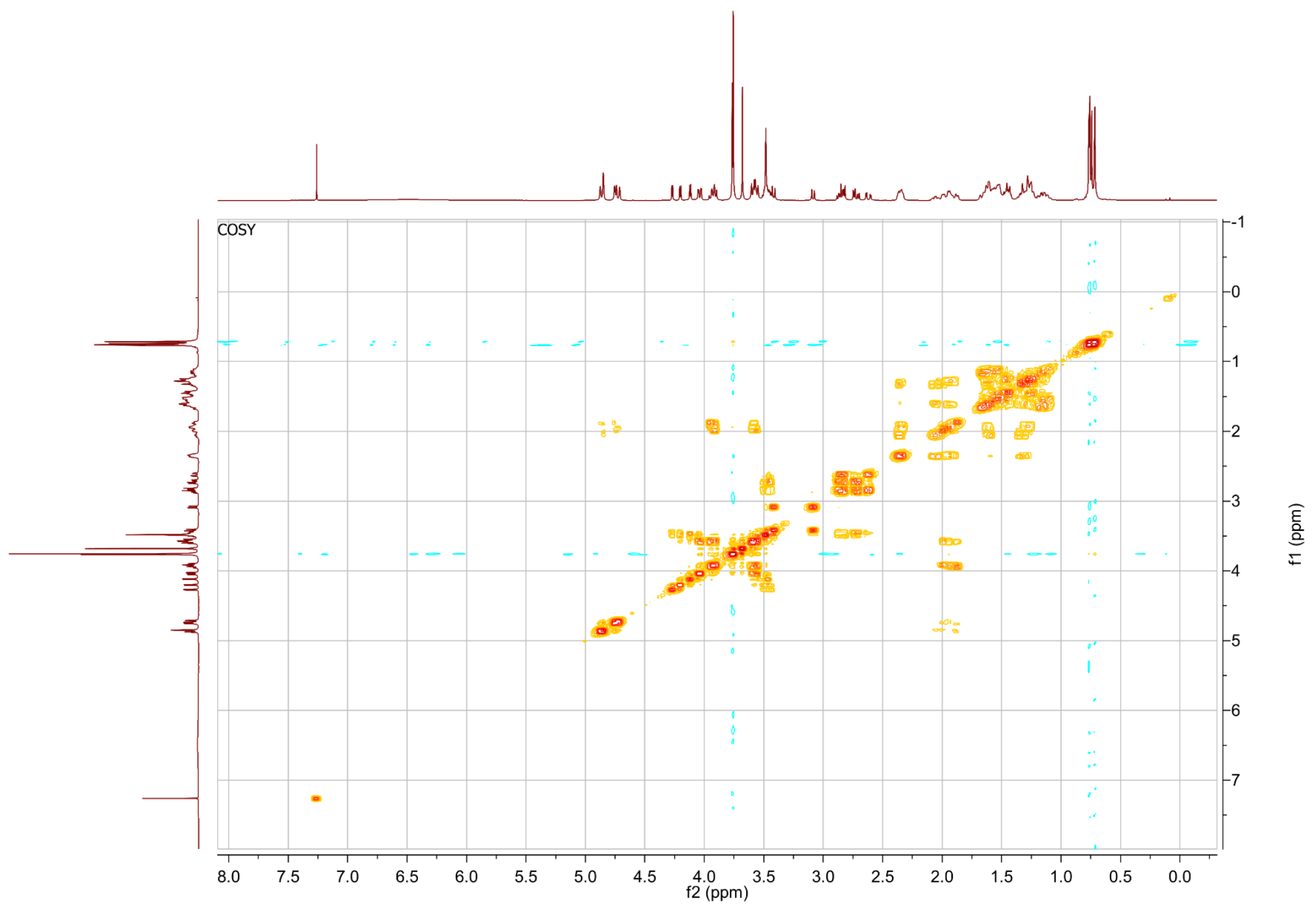

Figure S8. ${ }^{1} \mathrm{H}-{ }^{1} \mathrm{H}$ COSY Spectrum of Cryptoporic Acid T (1) $\left(500 \mathrm{MHz}\right.$, in $\left.\mathrm{CDCl}_{3}\right)$. 


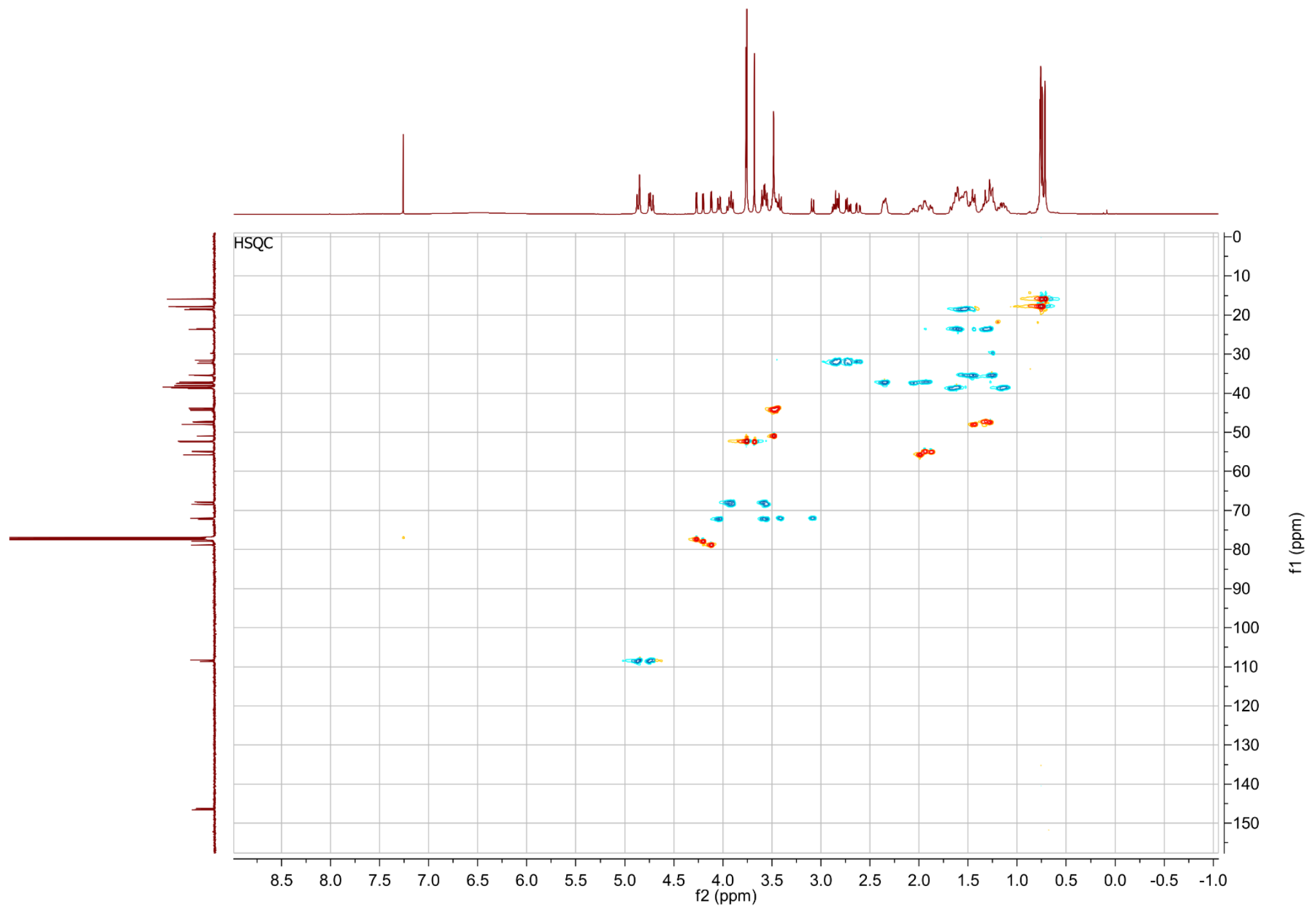

Figure S9. Multiplicity-edited HSQC Spectrum of Cryptoporic Acid T (1) (500 MHz, in $\mathrm{CDCl}_{3}$ ). 


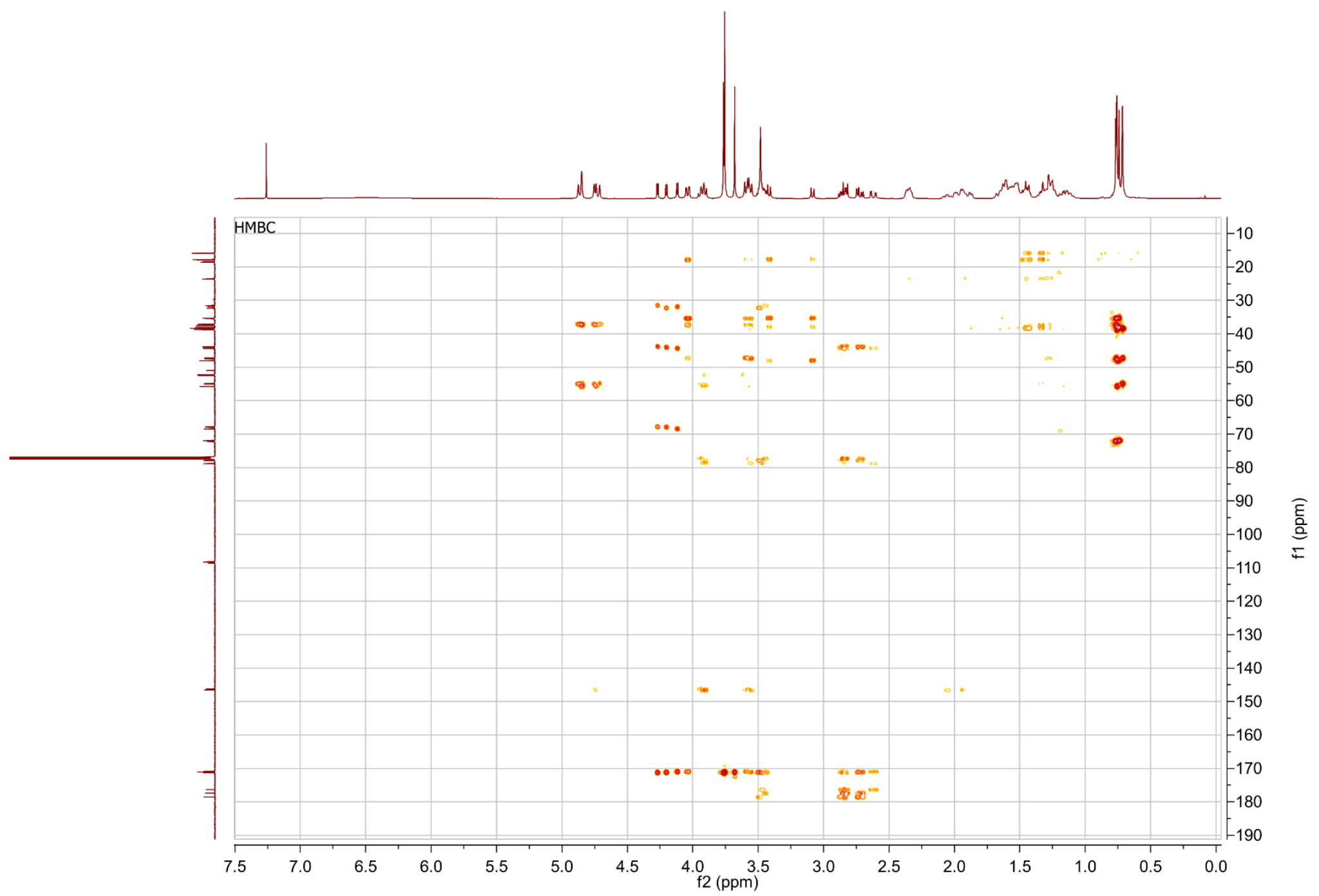

Figure S10. HMBC Spectrum of Cryptoporic Acid T (1) (500 MHz, in $\mathrm{CDCl}_{3}$ ). 


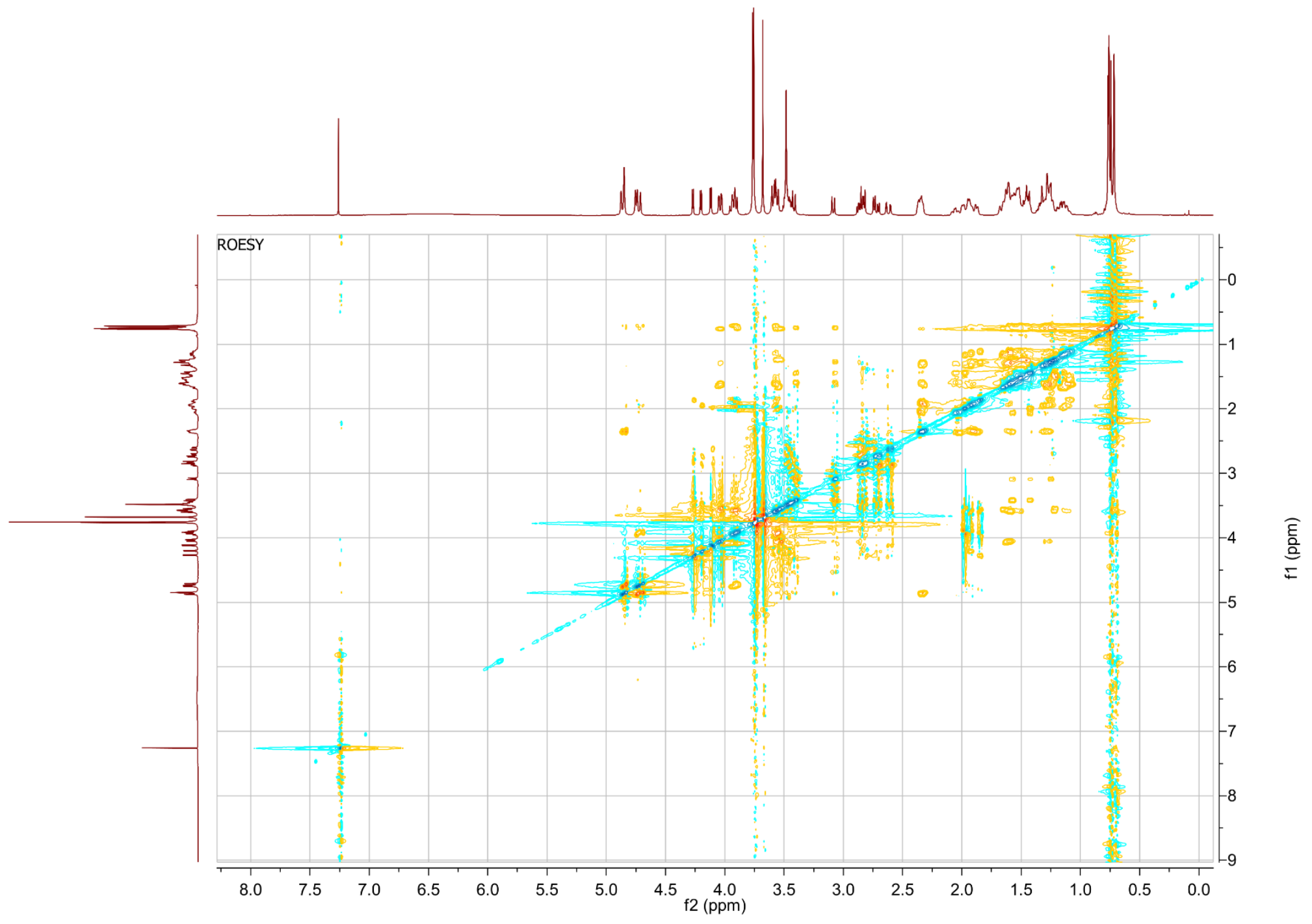

Figure S11. ROESY Spectrum of Cryptoporic Acid T (1) (500 MHz, in $\mathrm{CDCl}_{3}$ ). 


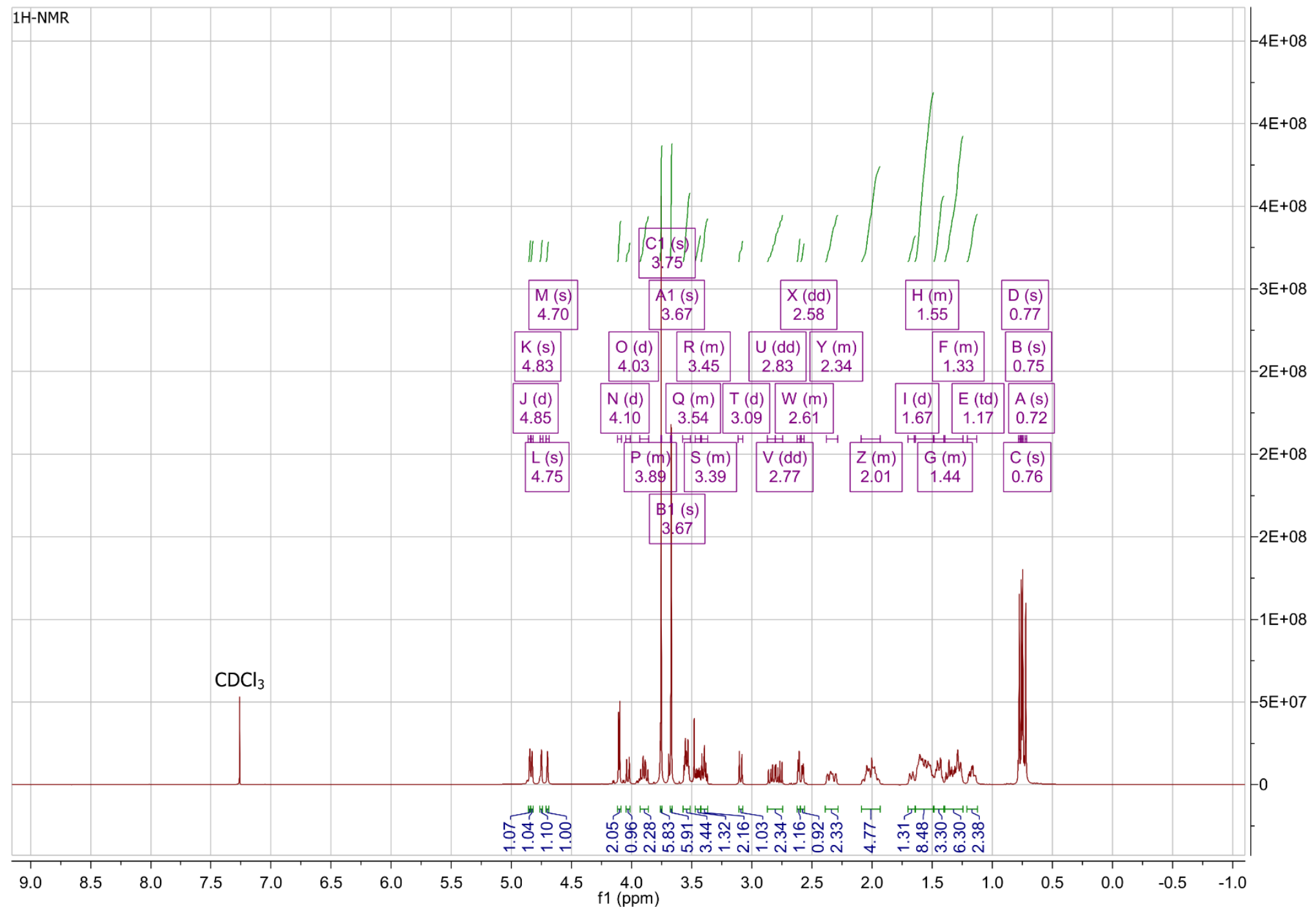

Figure S12. ${ }^{1} \mathrm{H}$ NMR Spectrum of 5"', 6 "'"-cryptoporic acid G dimethyl ester (2) (500 MHz, in $\mathrm{CDCl}_{3}$ ). 


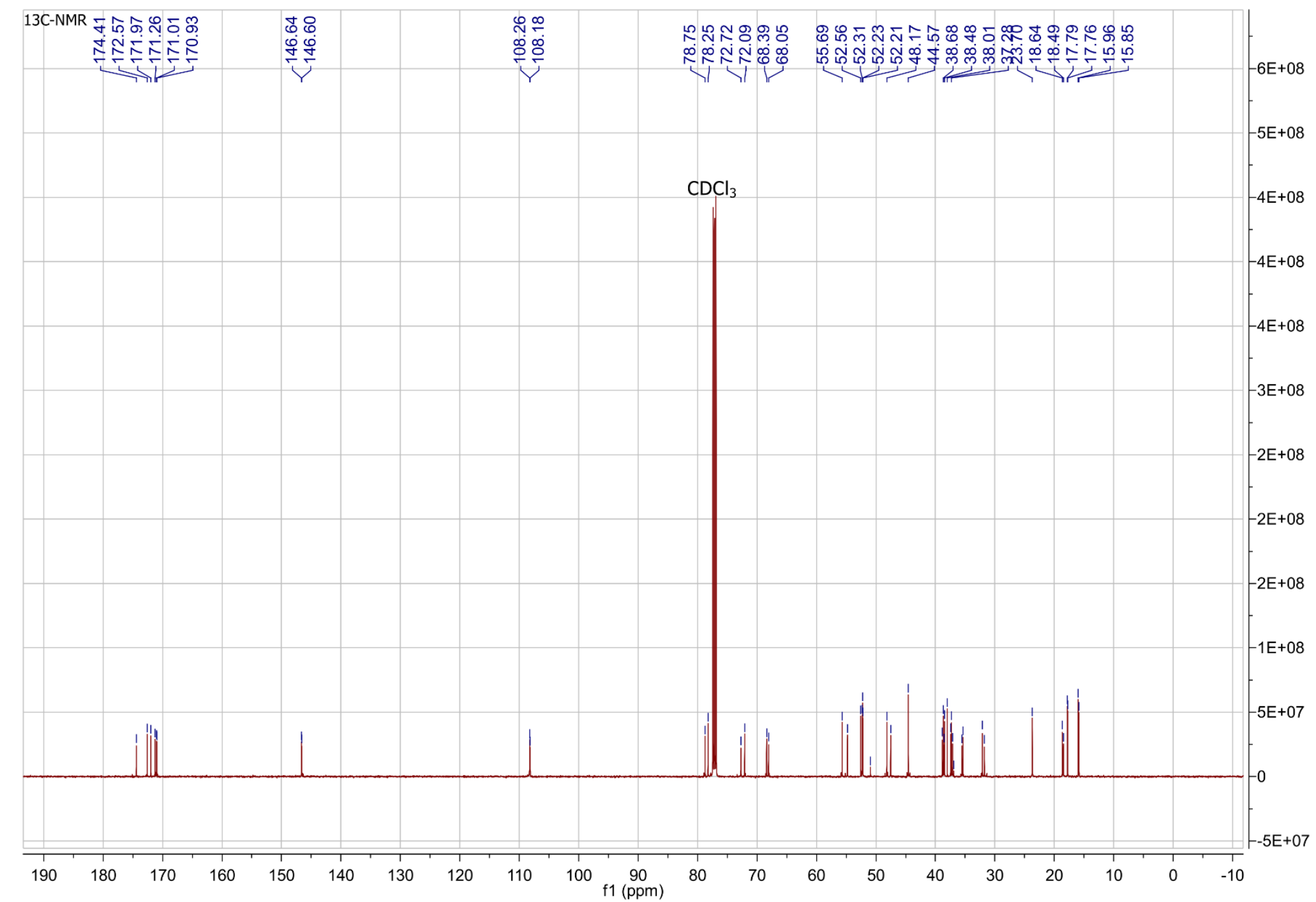

Figure S13. ${ }^{13} \mathrm{C}$ NMR Spectrum of 5"', 6"'-cryptoporic acid G dimethyl ester (2) (125 MHz, in $\mathrm{CDCl}_{3}$ ). 


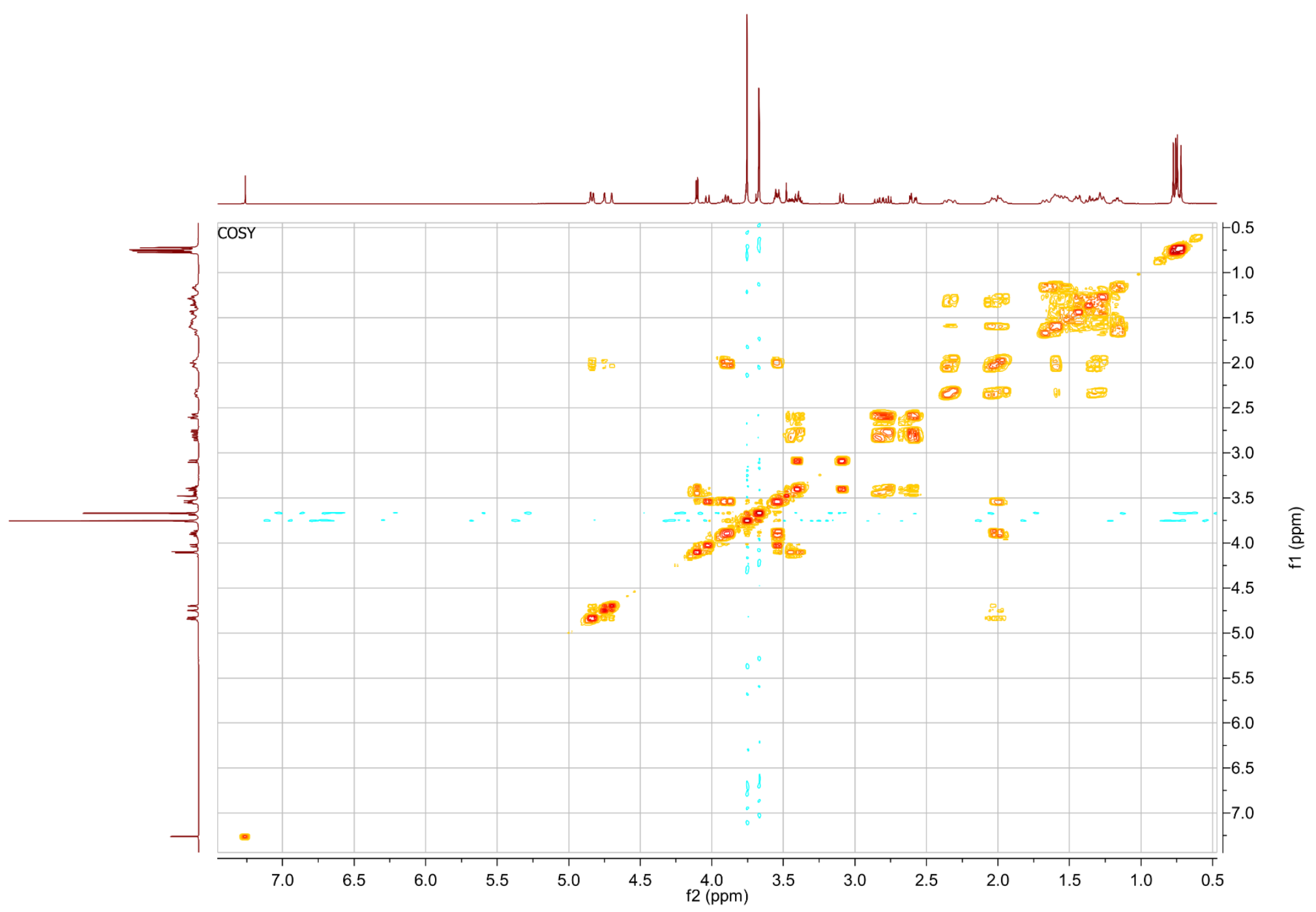

Figure S14. ${ }^{1} \mathrm{H}-{ }^{1} \mathrm{H}$ COSY Spectrum of $5^{\prime \prime \prime}, 66^{\prime \prime \prime}$-cryptoporic acid G dimethyl ester (2) (500 MHz, in $\mathrm{CDCl}_{3}$ ). 


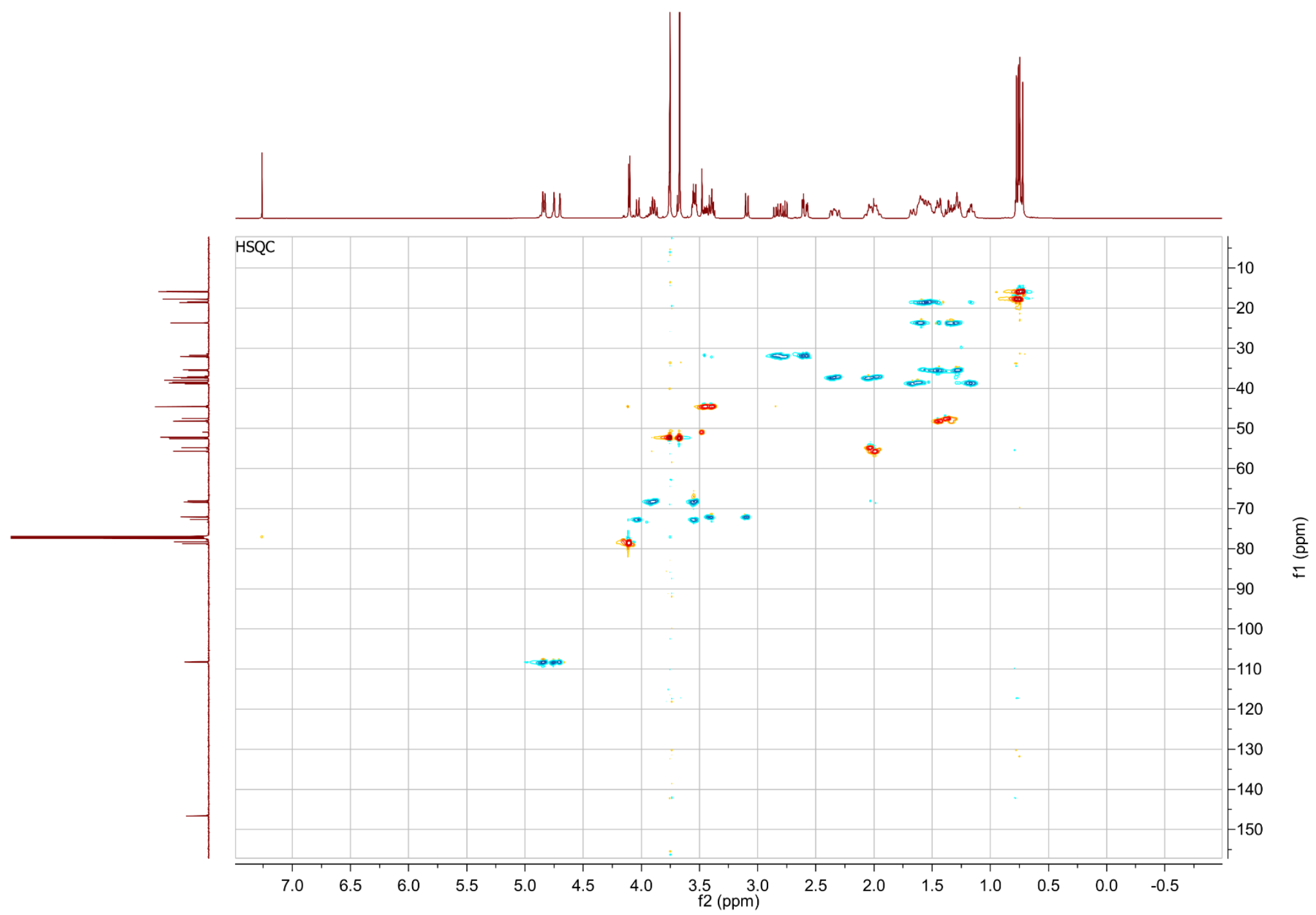

Figure S15. Multiplicity-edited HSQC Spectrum of 5"',6"'--cryptoporic acid G dimethyl ester (2) (500 MHz, in $\mathrm{CDCl}_{3}$ ). 


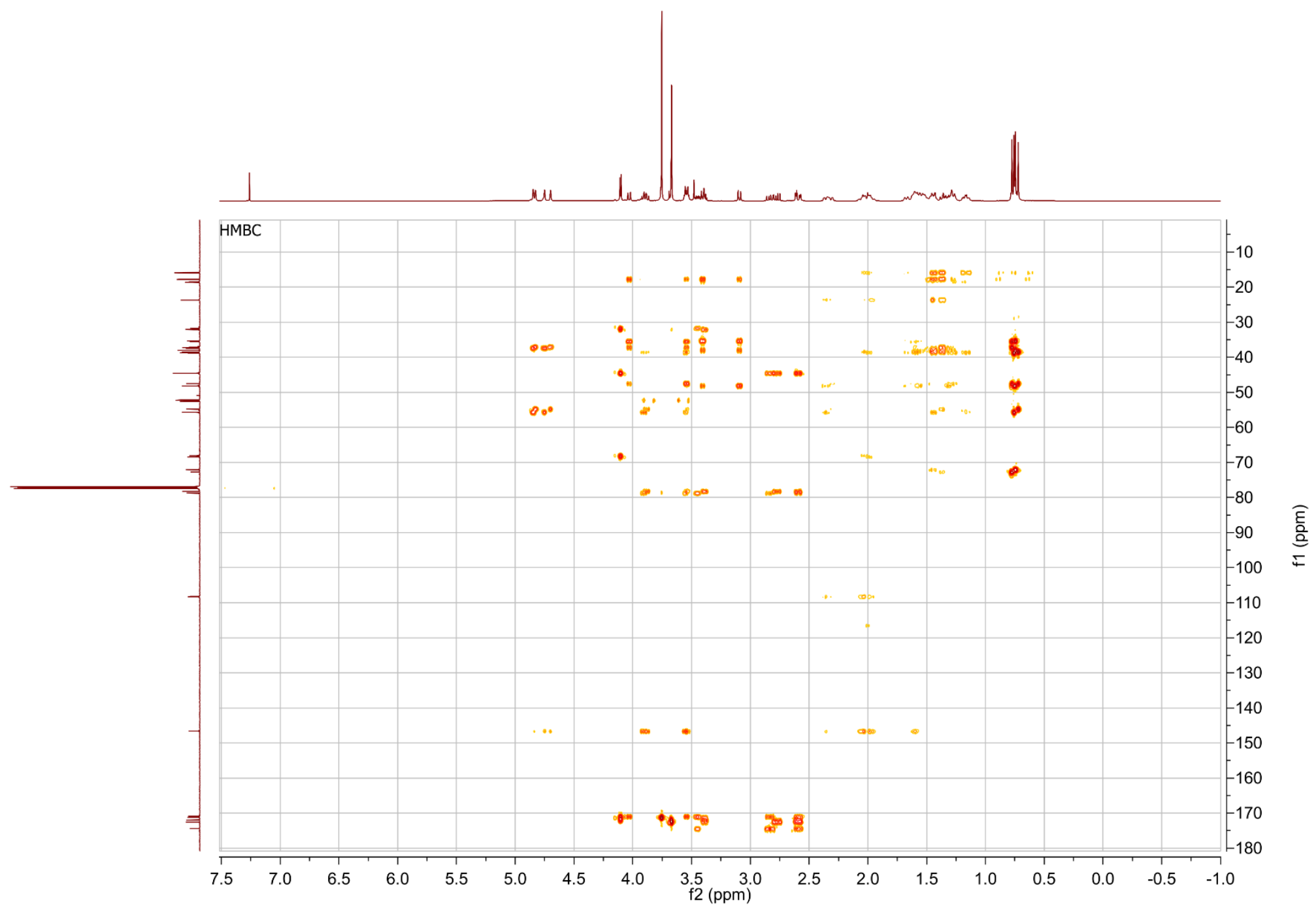

Figure S16. HMBC Spectrum of 5"',6"'--cryptoporic acid G dimethyl ester (2) (500 MHz, in $\mathrm{CDCl}_{3}$ ). 


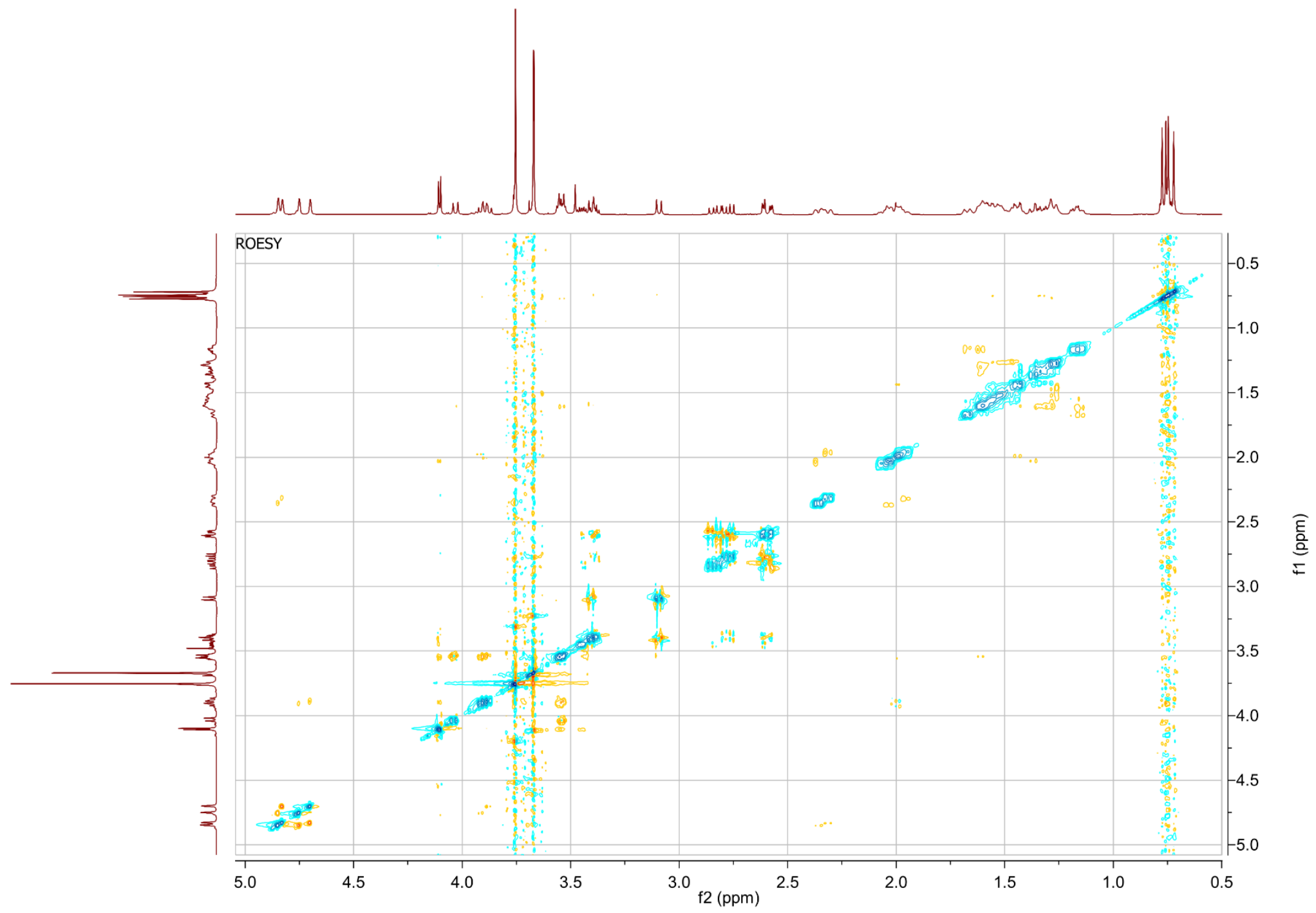

Figure S17. ROESY Spectrum of 5"', 6 "'--cryptoporic acid G dimethyl ester (2) (500 MHz, in $\mathrm{CDCl}_{3}$ ). 


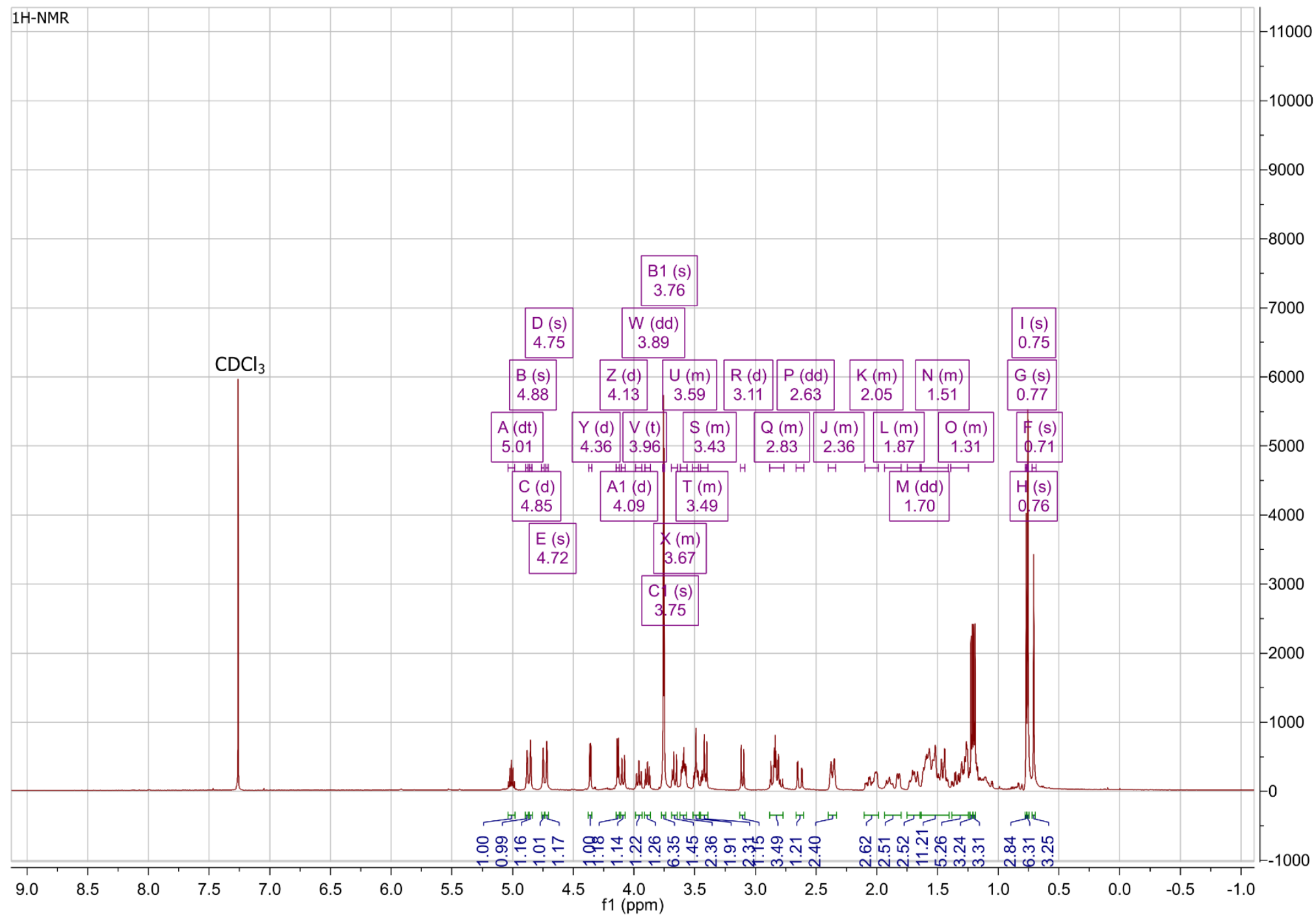


Figure S18. ${ }^{1} \mathrm{H}$ NMR Spectrum of 5 "-cryptoporic acid E isopropyl ester (3) (500 MHz, in $\mathrm{CDCl}_{3}$ ). 


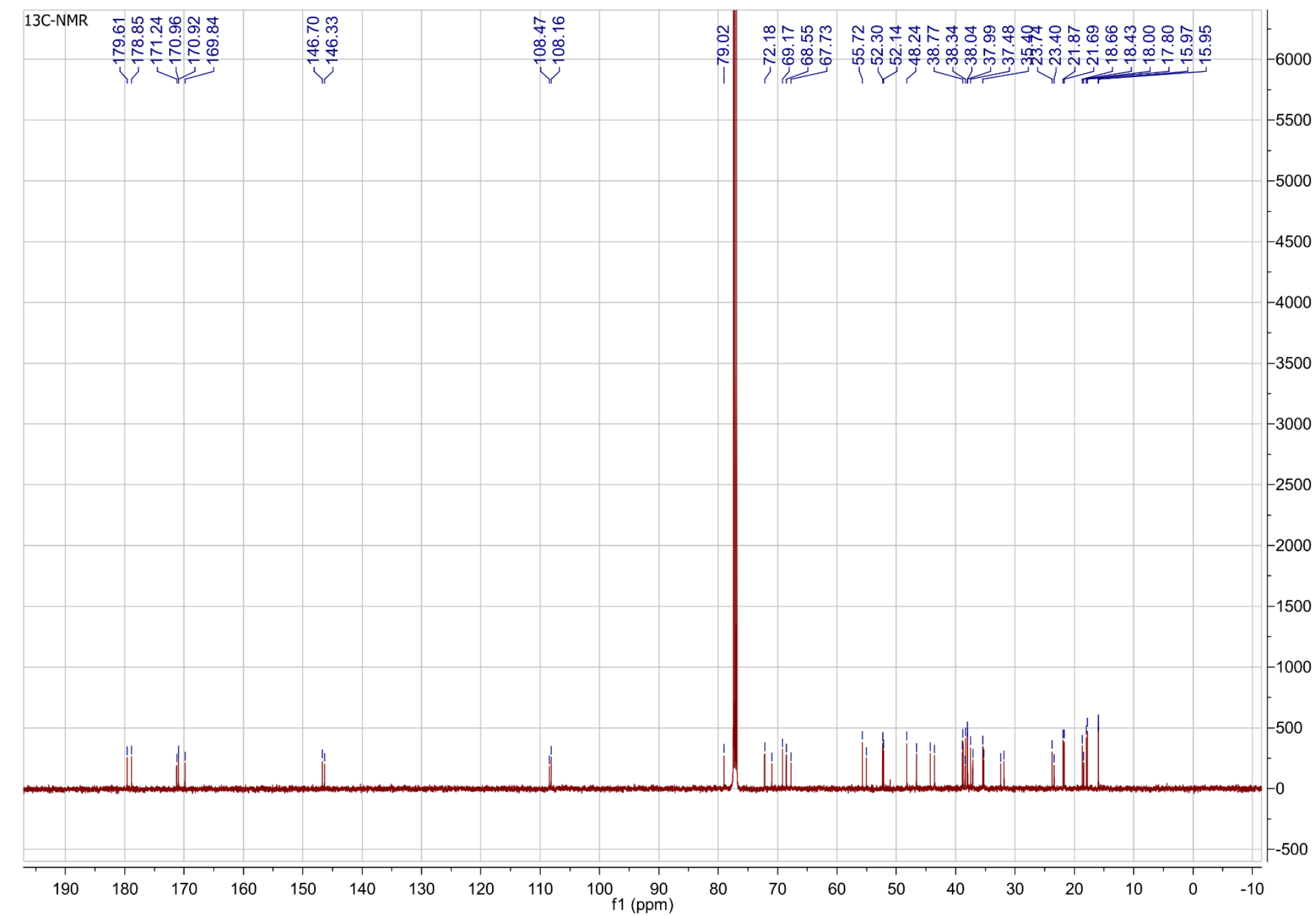


Figure S19. ${ }^{13} \mathrm{C}$ NMR Spectrum of 5"-cryptoporic acid E isopropyl ester (3) (125 MHz, in $\mathrm{CDCl}_{3}$ ). 


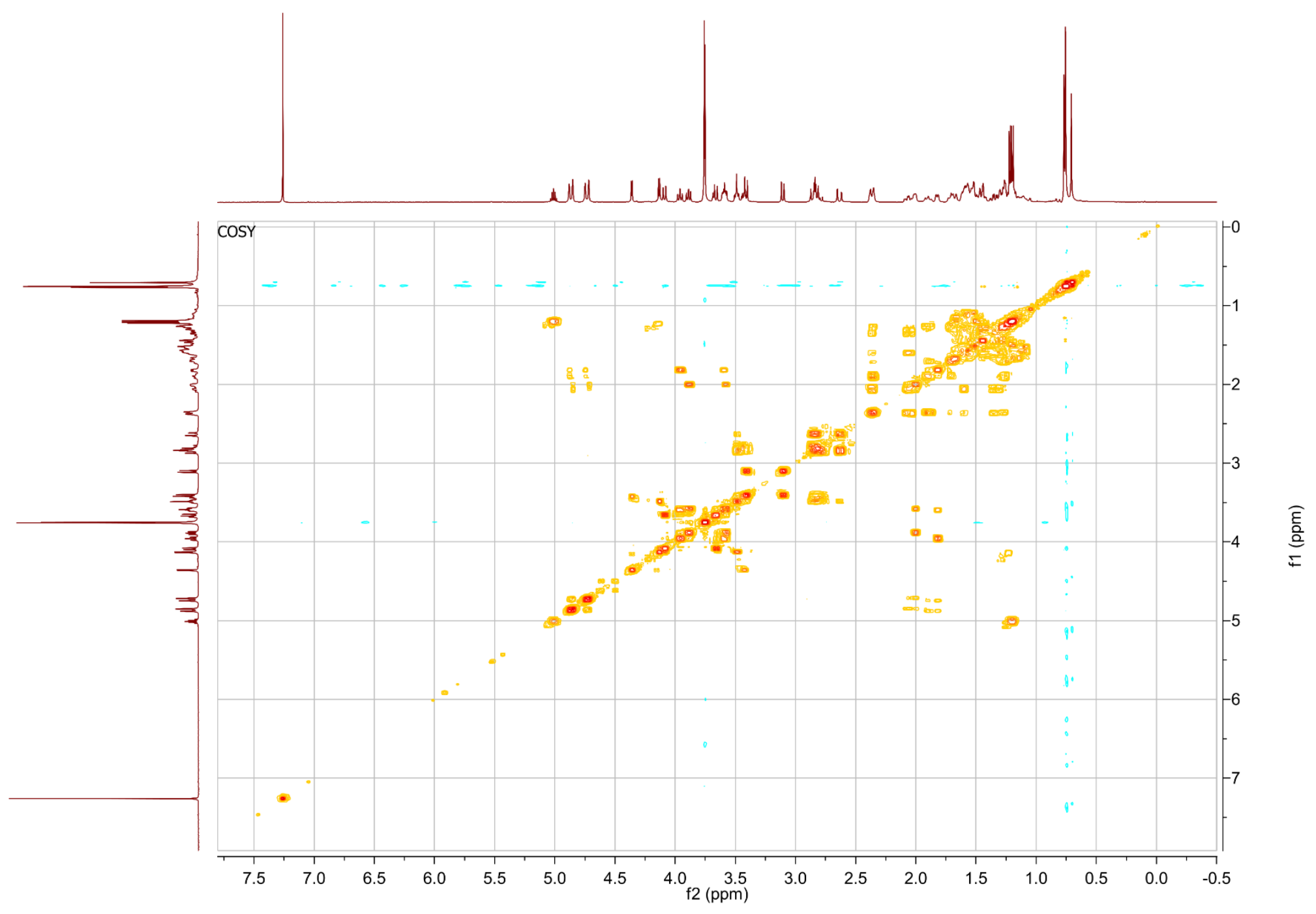


Figure S20. ${ }^{1} \mathrm{H}-{ }^{1} \mathrm{H}$ COSY Spectrum of 5 "-cryptoporic acid E isopropyl ester (3) (500 MHz, in $\mathrm{CDCl}_{3}$ ). 


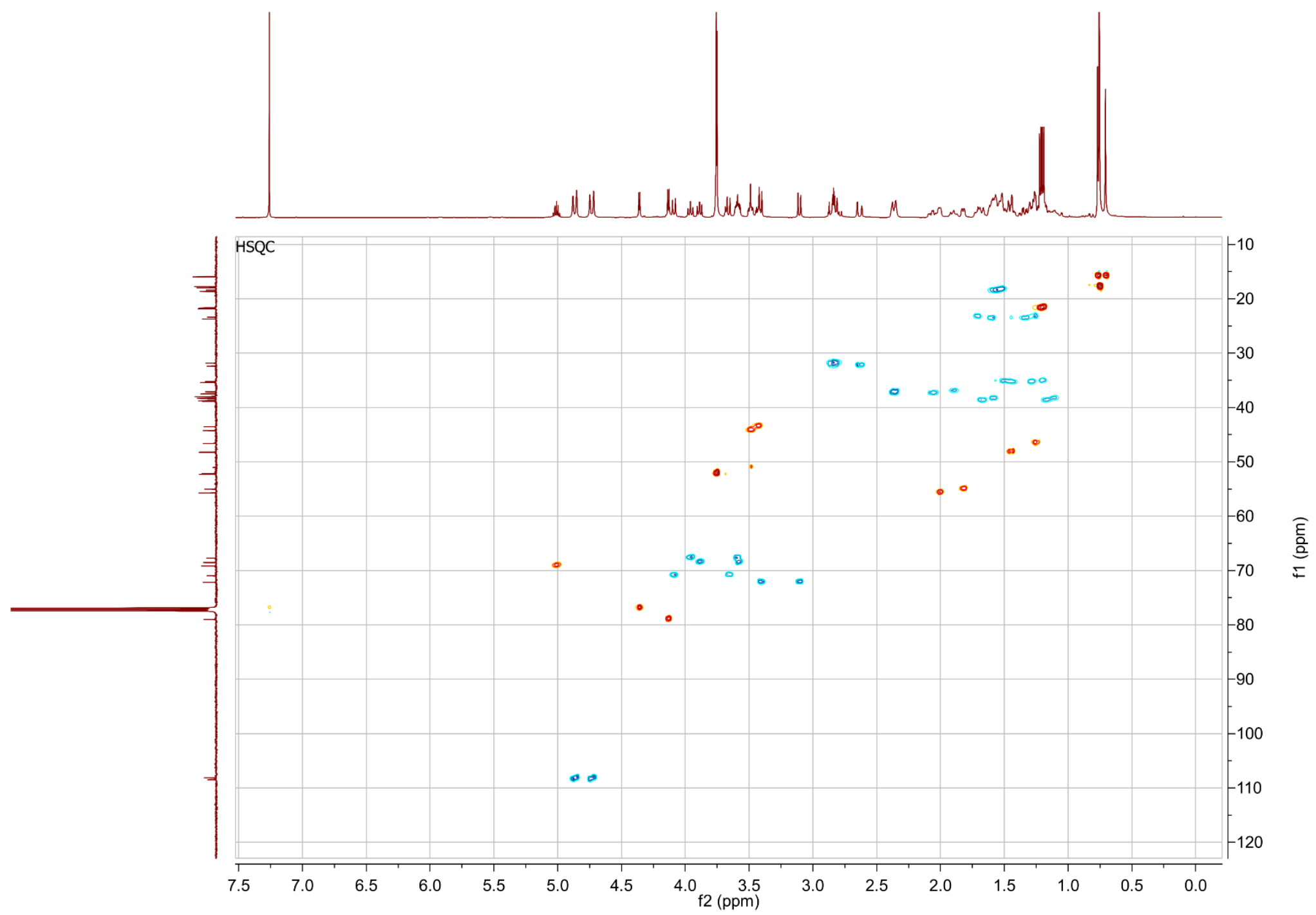


Figure S21. Multiplicity-edited HSQC Spectrum of 5"-cryptoporic acid E isopropyl ester (3) (500 MHz, in $\mathrm{CDCl}_{3}$ ). 


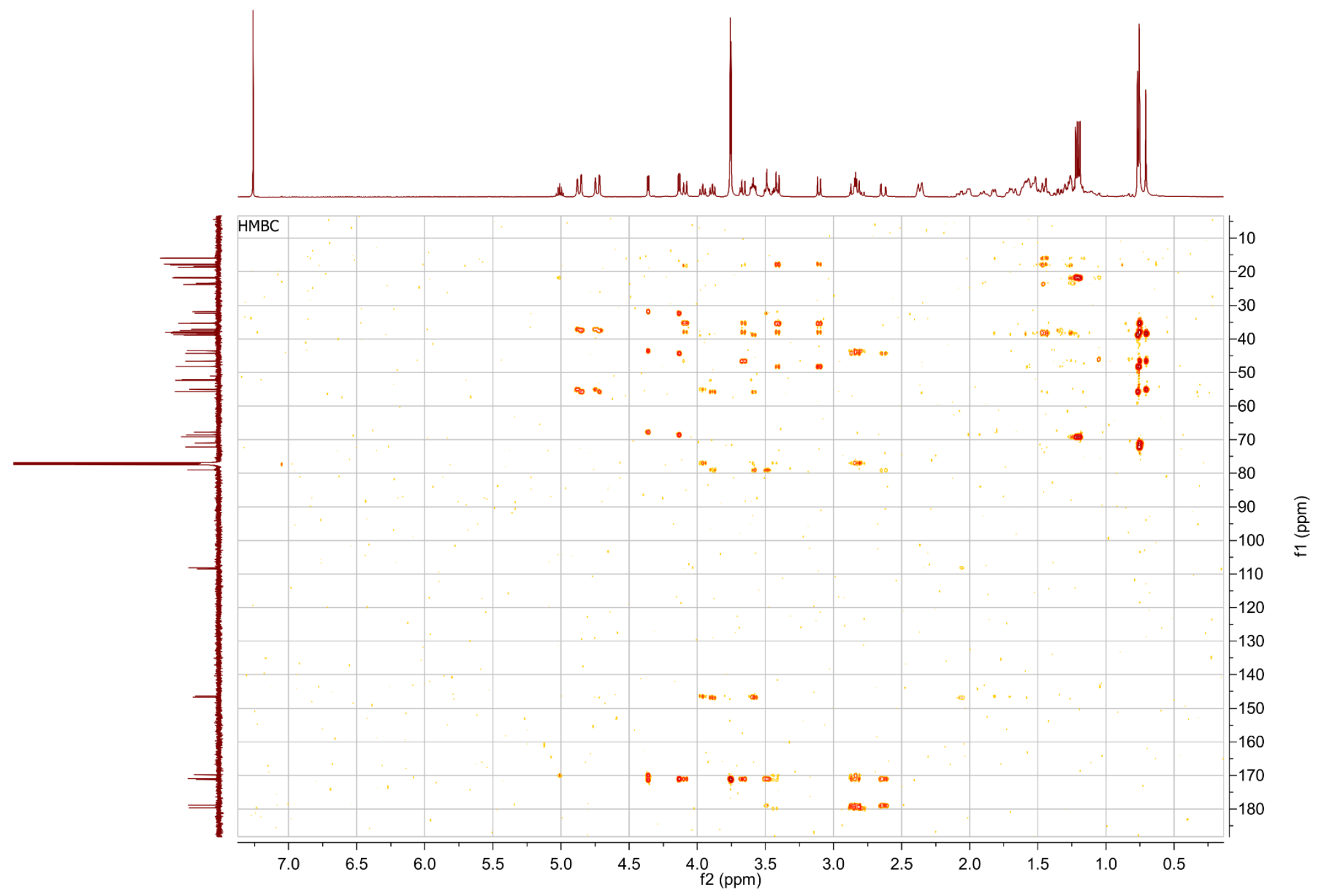


Figure S22. HMBC Spectrum of 5"-cryptoporic acid E isopropyl ester (3) (500 MHz, in $\mathrm{CDCl}_{3}$ ). 


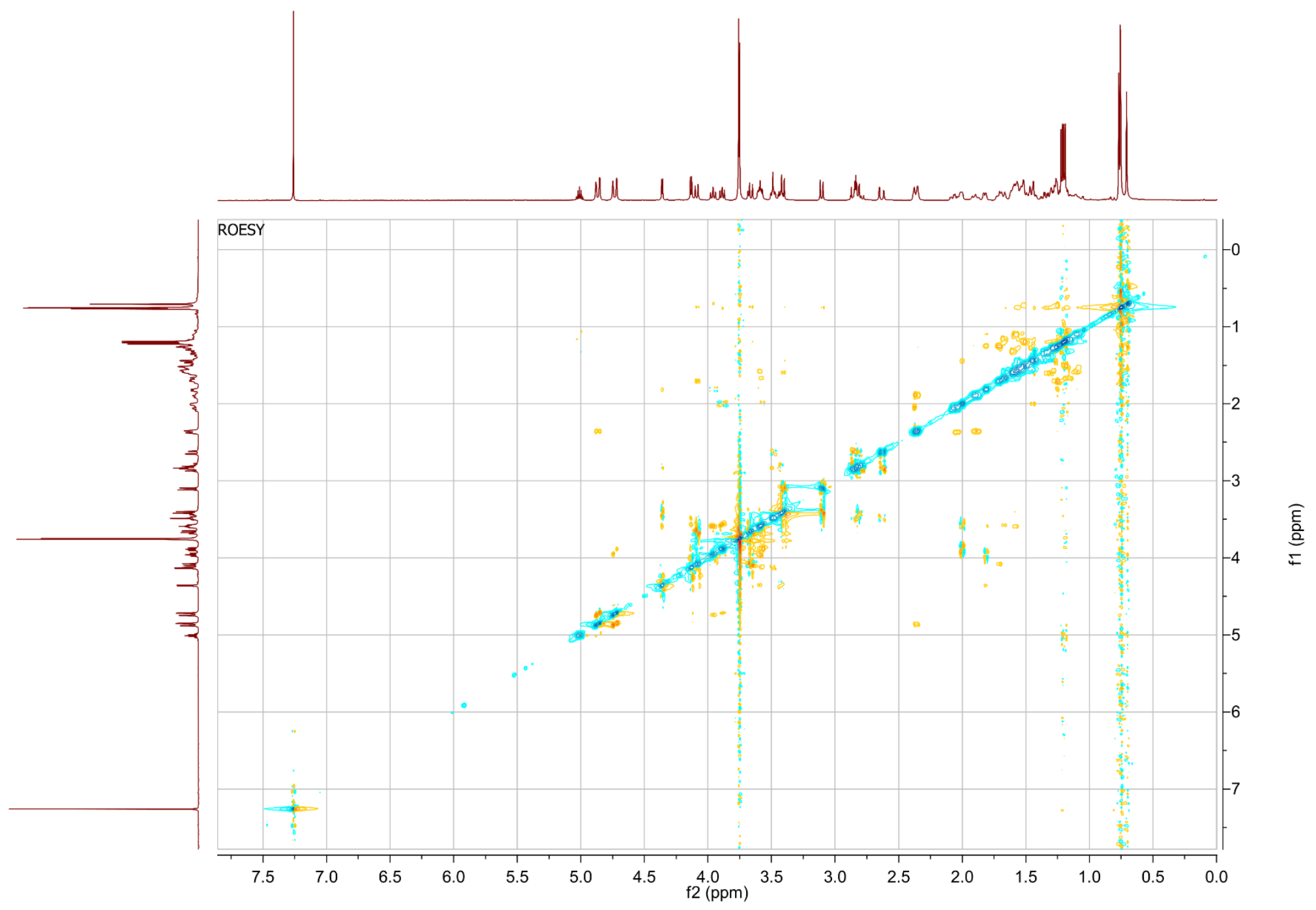


Figure S23. ROESY Spectrum of 5"-cryptoporic acid E isopropyl ester (3) (500 MHz, in $\mathrm{CDCl}_{3}$ ). 


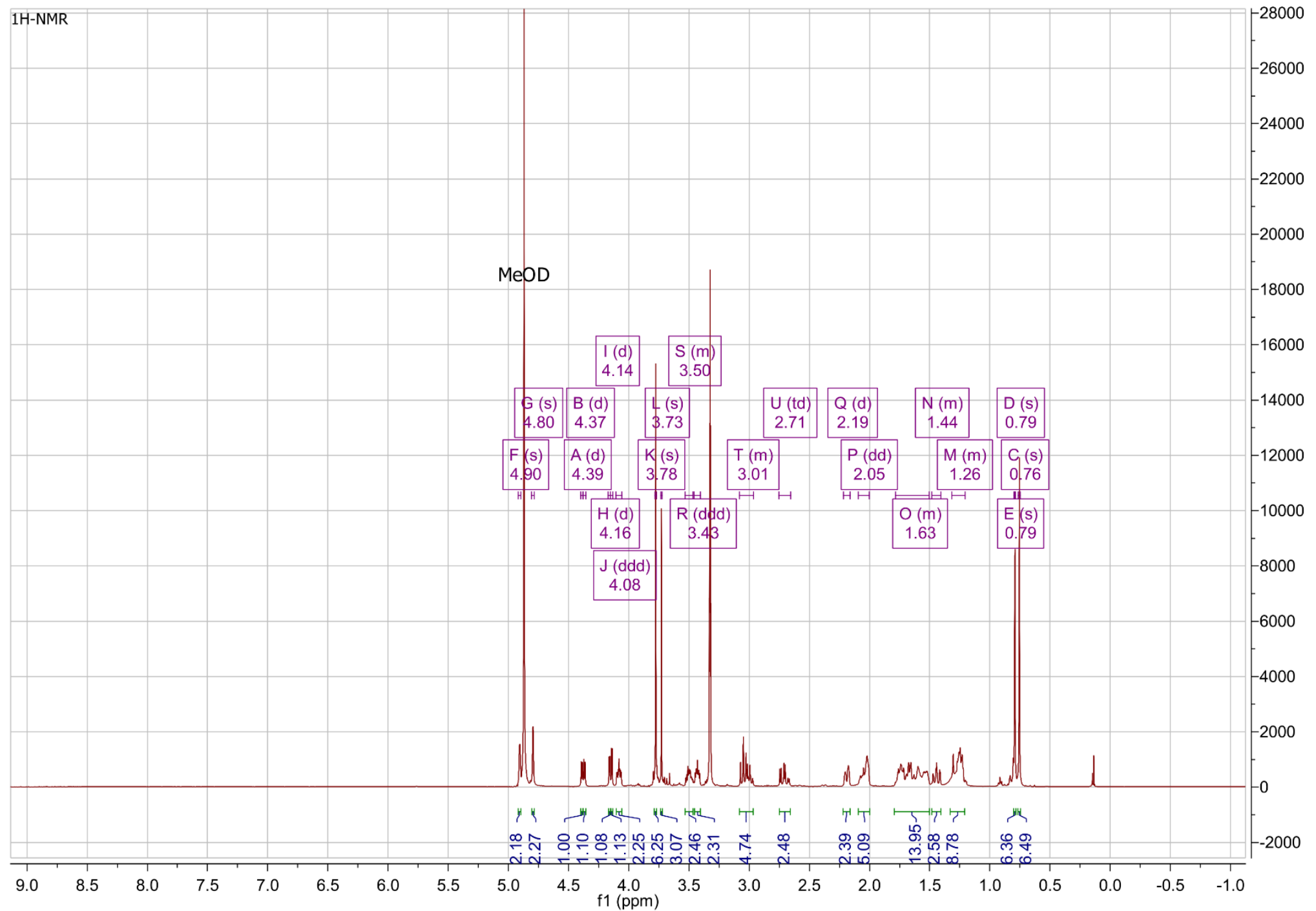

Figure S24. ${ }^{1} \mathrm{H}$ NMR Spectrum of Cryptoporic acid D trimethyl ester (4) (500 MHz, in MeOD). 


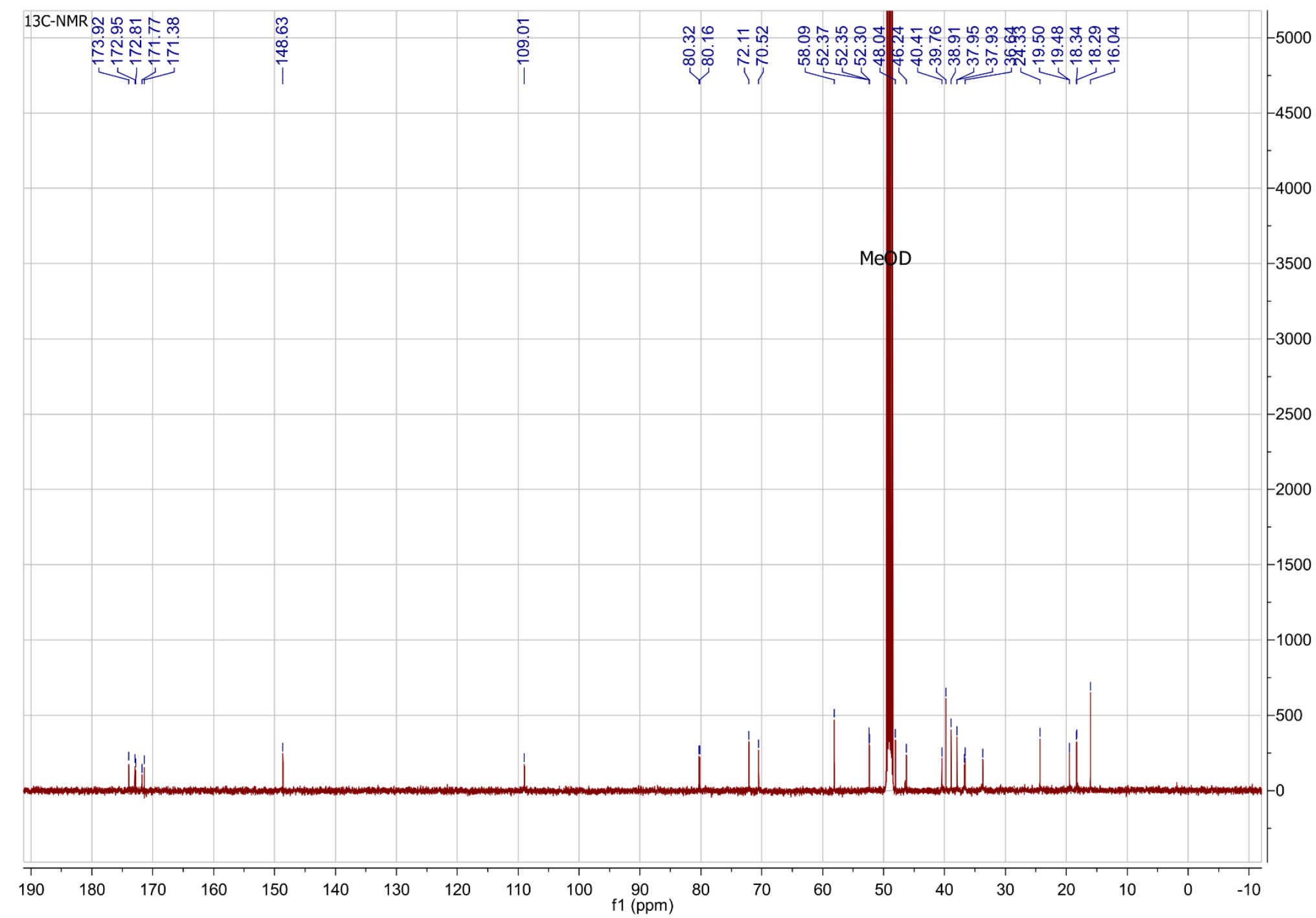

Figure S25. ${ }^{13} \mathrm{C}$ NMR Spectrum of Cryptoporic acid D trimethyl ester (4) (125 MHz, in MeOD). 


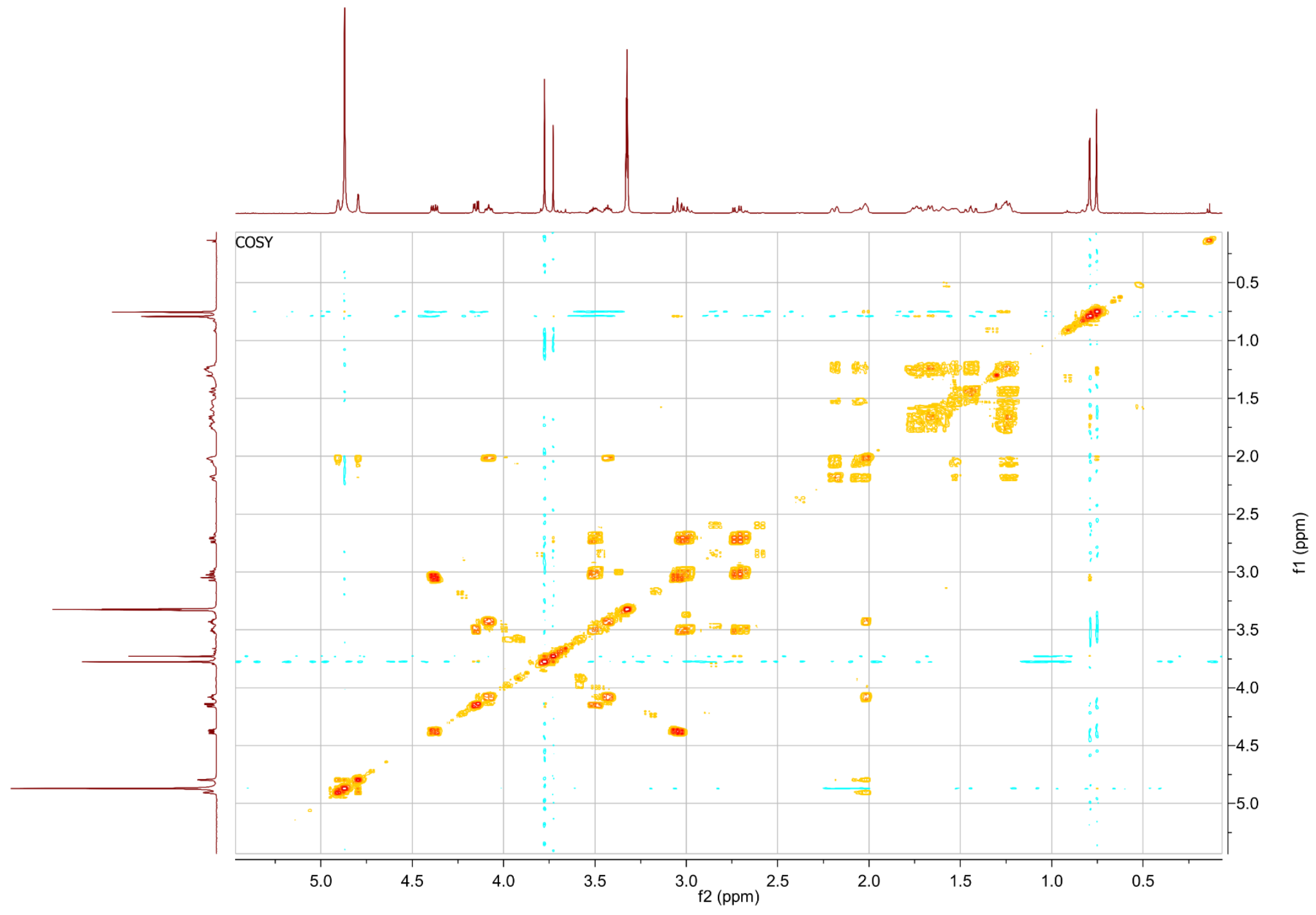

Figure S26. ${ }^{1} \mathrm{H}-{ }^{1} \mathrm{H}$ COSY Spectrum of Cryptoporic acid D trimethyl ester (4) (500 MHz, in MeOD). 


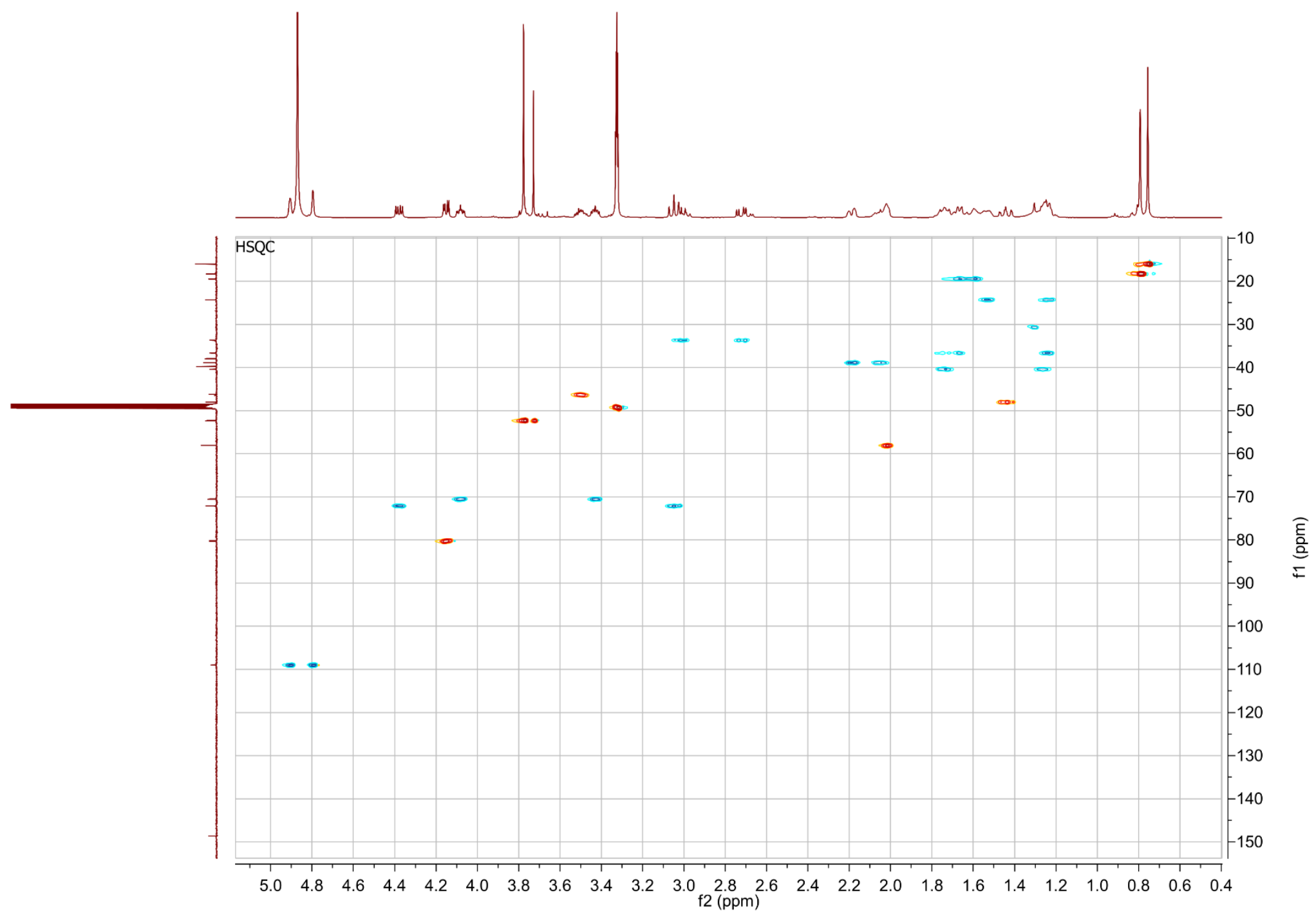

Figure S27. Multiplicity-edited HSQC Spectrum of Cryptoporic acid D trimethyl ester (4) (500 MHz, in MeOD). 


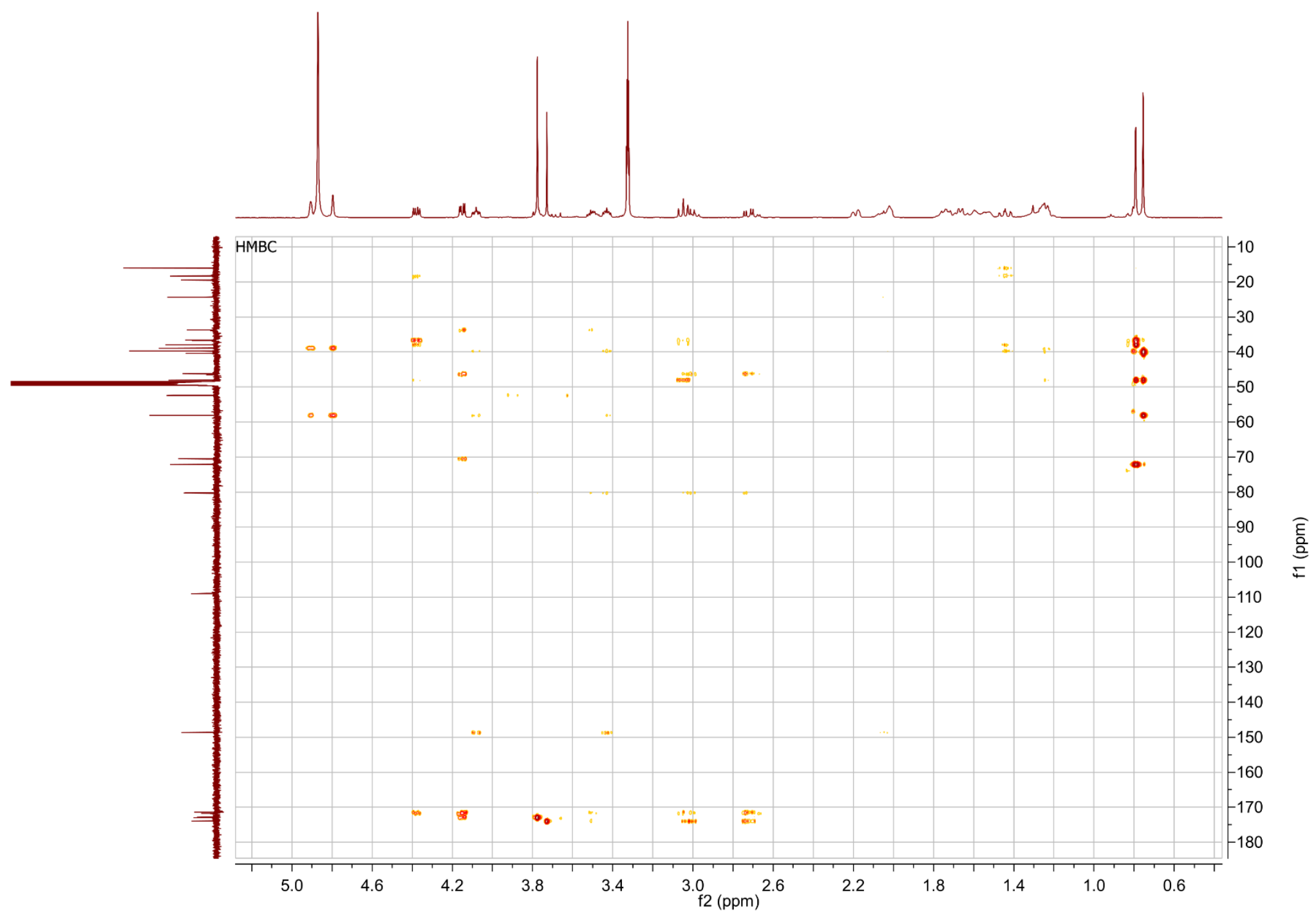

Figure S28. HMBC Spectrum of Cryptoporic acid D trimethyl ester (4) (500 MHz, in MeOD). 